\title{
含双酰胺结构的氟吡菌酰胺衍生物的设计、合成及生物活性研究
}

\author{
许庆博梁培博路慧哲 金淑惠* 董燕红张建军* \\ (中国农业大学理学院 北京 100193)
}

\begin{abstract}
摘要 基于活性亚结构拼接法, 将双酰胺结构引入氟吡菌酰胺的骨架中, 设计并合成了 22 个含双酰胺结构的氟吡菌酰 胺衍生物. 结构经过 ${ }^{1} \mathrm{H} N \mathrm{NR} 、{ }^{13} \mathrm{C}$ NMR 和 HRMS 鉴定，目标化合物对 4 种植物病原真菌离体的抑制活性以及对秀丽 隐杆线虫的离体杀虫活性也进行了研究. 结果显示, 在 $50 \mathrm{mg} / \mathrm{L}$ 的浓度下, 大部分化合物对苹果腐烂病原菌及油菜菌核 病原菌有较高的抑制活性, 抑制率在 80\%以上. 其中 $N$-(2-(3-氯-5-(三氟甲基)吡啶-2-基)乙基)-2-(3-氟苯甲酰胺基)苯甲 酰胺(7f) 对苹果腐烂病原菌的 $\mathrm{EC}_{50}$ 为 $6.12 \mathrm{mg} / \mathrm{L}$, 高于对照药剂氟吡菌酰胺 $(35.5 \mathrm{mg} / \mathrm{L})$; 对苹果腐烂病原菌 $\mathrm{SDH}$ 酶抑制 活性在 $100 \mu \mathrm{mol}$ 的浓度下达到 $90.1 \%$; 对秀丽隐杆线虫的致死率在 $200 \mathrm{mg} / \mathrm{L}$ 浓度下为 $87 \%$. 分子对接结果表明, SDH 可能是化合物的潜在靶标.
\end{abstract}

关键词＼cjkstart琥珀酸脱氢酶; 杀线虫剂; 双酰胺; 氟吡菌酰胺

\section{Design, Synthesis and Bioactivity of Fluopyram Derivatives Containing Diamide Moiety}

\author{
$\mathrm{Xu}$, Qingbo Liang, Peibo Lu, Huizhe Jin, Shuhui* \\ Dong, Yanhong Zhang, Jianjun* \\ (College of Science, China Agricultural University, Beijing 100193)
}

\begin{abstract}
According to the principle of combination of bioactive substructure, the structure of diamide was introduced into fluopyram, and 22 novel fluopyram derivatives containing diamide were designed and synthesized. The structures of the target compounds were characterized by ${ }^{1} \mathrm{H}$ NMR, ${ }^{13} \mathrm{C}$ NMR and HRMS. The inhibitory activities of the target compounds against four plant pathogenic fungi and in vitro insecticidal activities against Caenorhabditis elegans were studied. The results showed that under the concentration of $50 \mathrm{mg} / \mathrm{L}$, most of the compounds showed moderate fungicide activity against Cytospora sp. and Sclerotinia sclerotiorum with inhibition rate over $80 \%$. The $\mathrm{EC}_{50}$ of $N$-(2-(3-chloro-5-(trifluoromethyl)pyridin-2-yl)ethyl)2-(3-fluorobenzamido)benzamide (7f) to Cytospora sp. was $6.12 \mathrm{mg} / \mathrm{L}$, which is higher than fluopyram (35.5 mg/L), the SDH essay showed that enzyme inhibition rate of $7 \mathbf{f}$ reached $90.1 \%$, and the mortality rate against C. elegans was $87 \%$ at $200 \mathrm{mg} / \mathrm{L}$. The results of molecular docking show that the SDH may be a potential target of the compounds.
\end{abstract}

Keywords succinate dehydrogenase; nematicide; diamide; fluopyram

氟吡菌酰胺(Fluopyram, 图 1B)是由拜尔作物科学 公司开发, 于 2010 年在中国获得临时登记用于黄瓜白 粉病防治, 可对灰霉病菌、白粉病菌、核盘菌属以及从 梗孢属病菌有较好的防治效果; 2012 年在中国登记了 41.7\% 氟吡菌酰胺制剂农药(PD: 20121664, 注册商标: 路富达), 用于线虫防治 ${ }^{[1-3]}$. 氟吡菌酰胺(路富达)作为一 种新型的杀线虫剂, 作用机制为抑制靶标线粒体呼吸链 复合体 II(琥珀酸脱氢酶, $\mathrm{SDH}^{[4]}$ ) 的活性, 从而干扰线虫
的呼吸作用，使其虫体僵直，活动力急剧下降，进而导 致线虫死亡. 氟吡菌酰胺(路富达)也是第一个通过抑制 琥珀酸脱氢酶的杀线虫剂，因其具有低毒、环保、高效、 多功能性等优点，有望成为线虫防治战略中的支柱产 品.

琥珀酸脱氢酶是三羧酸(TCA)循环中唯一整合在膜 上的多亚基酶 ${ }^{[5]}$. 在真核生物中，它结合在线粒体的内 膜上; 而在原核生物中, 它整合在细胞膜上. 它是连接

\footnotetext{
*Corresponding authors. E-mail: shuhuij@cau.edu.cn; zhangjianjun@cau.edu.cn Received March 10, 2021; revised April 7, 2021; published online June 1, 2021. Project supported by the National Key R\&D Program of China (No. 2018YFD0200100) and the National Natural Science Foundation of China (No. 21772230).

国家重点研发计划(No. 2018YFD0200100)和国家自然科学基金(No. 21772230 )资助项目.
} 


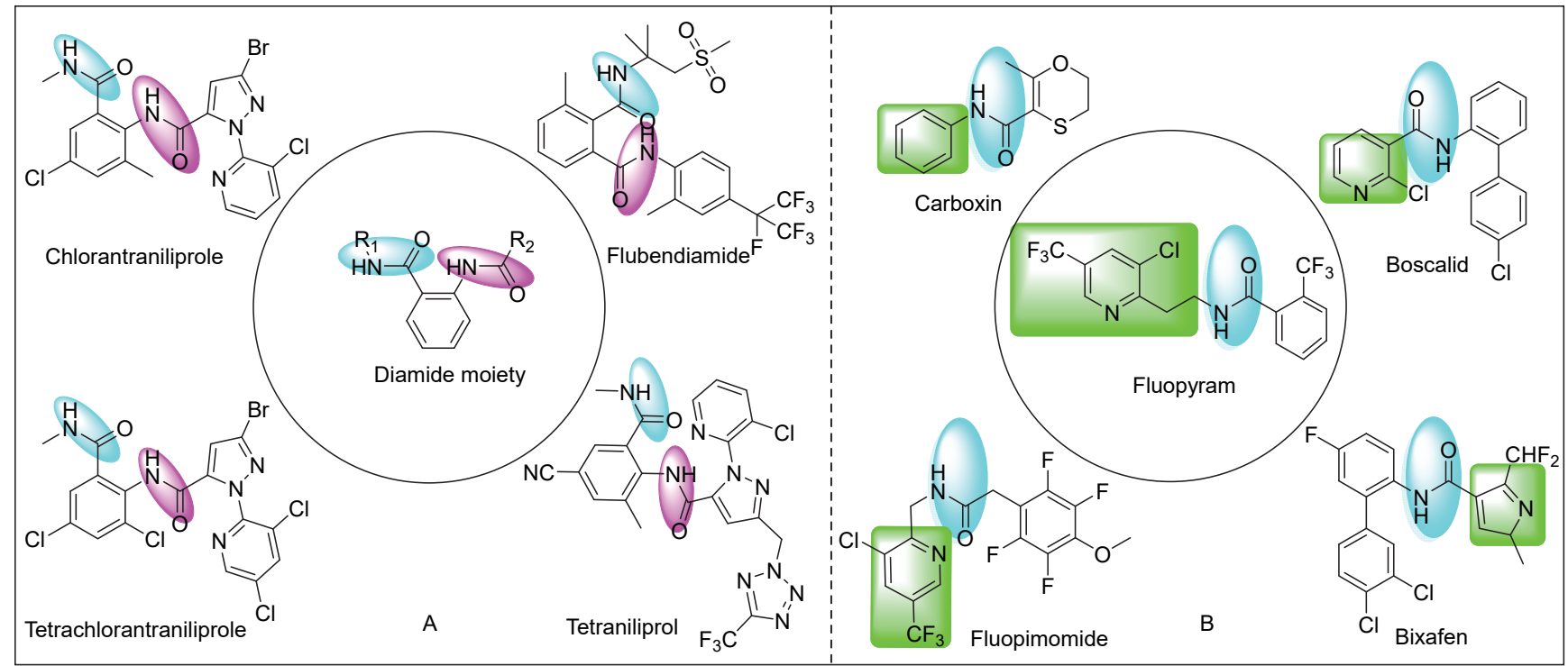

图 1 部分双酰胺类杀虫剂及 SDHI 类杀菌剂

Figure 1 Structures of diamide pesticide and fungicide

氧化磷酸化和电子转移的枢纽之一 ${ }^{[6-8]}$, 可以为真核细 胞线粒体、各种原核细胞的需氧及产能呼吸链提供电子. 琥珀酸脱氢酶抑制剂(SDHI)通过抑制呼吸链电子传递, 从而破坏线粒体的三羧酸循环, 进而导致线粒体不能够 产生能量, 最终导致病原体死亡 ${ }^{[9-12]}$. 自 20 世纪 60 年代 第一个琥珀酸脱氢酶抑制剂萎锈灵上市以来, 已有 23 种以 SDH 为靶标的杀菌剂陆续上市(如图 1B 所示), 其 销售额在农药杀菌剂领域占据重要比重, 这些杀菌剂在 分子结构上均具有酰胺键. 其中, 氟吡菌酰胺因兼具良 好的杀菌以及杀线虫活性, 受到了广泛的关注. 因此, 近些年来以氟吡菌酰胺为先导化合物开展靶向 SDH 酶 的杀菌剂和杀线虫剂的分子设计, 成为了研究热点.

双酰胺衍生物在医药和农药中具有重要作用 ${ }^{[13]}$, 它们具有广谱的生物活性, 如抗细菌 ${ }^{[14]}$ 、抗真菌 ${ }^{[15-17] 、}$ 除草、杀虫等活性 ${ }^{[18]}$. 如氯虫苯甲酰胺(Chlorantraniliprole, 图 1A) 是一种靶向鱼尼丁受体的高效广谱杀虫 剂. 根据前期对氟吡菌酰胺与靶酶的结合方式研究发 现, SDH 具有 “L” 型 “长” 的疏水的活性口袋, 吡定环 与 Trp67 形成 $\pi-\pi$ 相互作用, 酰胺的氧与 Pro2 11 形成氢 键相互作用. 为了寻找结构新颖的杀菌杀线虫小分子先 导化合物, 本文以氟吡菌酰胺为骨架, 保留氟吡菌酰胺 的吡啶与酰胺键片段, 结合双酰胺结构衍生物良好生物 活性, 通过亚结构拼接方法, 设计并合成了一种新型的 含双酰胺结构的氟吡菌酰胺衍生物(图 2).

\section{1 结果与讨论}

\section{1 化学合成}

设计的目标化合物合成过程如 Scheme 1 所示. 首
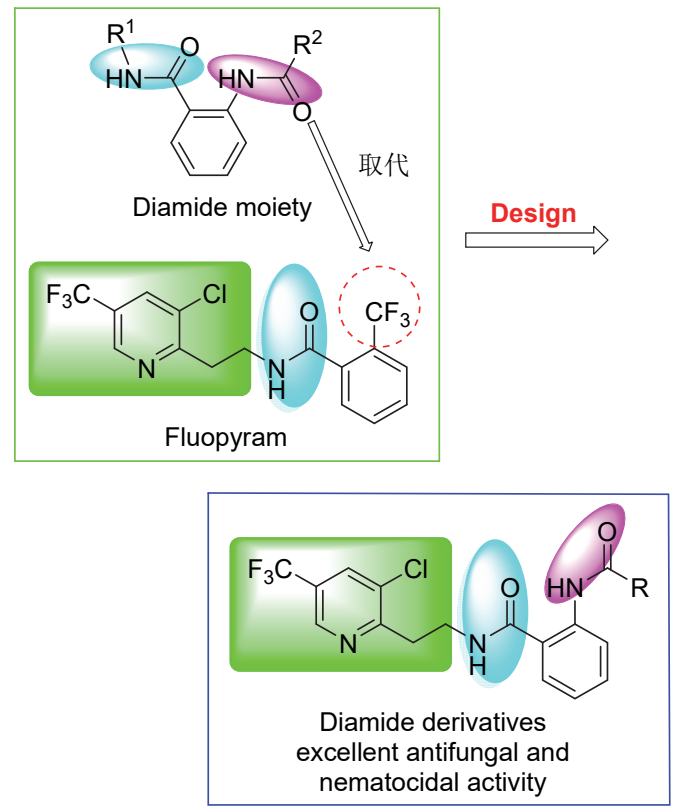

图 2 目标化合物的设计

Figure 2 Design of the title compounds

先以 2,3-二氯-5-三氟甲基-吡啶(1)为起始原料, 将其溶 于二甲基亚砜(DMSO)中, 以无水碳酸钾作碱, 氰基乙 酸乙酯作亲核试剂, 在 $70{ }^{\circ} \mathrm{C}$ 反应 $6 \mathrm{~h}$ 后经薄层色谱 (TLC)检测反应完成, 反应液冷却至室温后, 用稀盐酸 溶液调节 $\mathrm{pH}$ 为 2 , 有大量黄色固体析出, 过滤收集滤 饼, 得到化合物 $\mathbf{2}$.

参考文献[19]将化合物 2 溶于 $N, N$-二甲基甲酰胺 (DMF) 中, 用稀盐酸溶液调节 $\mathrm{pH}$ 为 2 , 采用温度为 $130{ }^{\circ} \mathrm{C}$, 并且加入大量 DMF, 导致后处理困难. 本文采 用水和 DMF 作混合溶剂, 在 $125{ }^{\circ} \mathrm{C}$ 下反应 $4 \mathrm{~h}$, 经 TLC 检测反应结束, 冷却至室温, 用 $4 \mathrm{~mol} / \mathrm{L}$ 的 $\mathrm{NaOH}$ 溶液调 


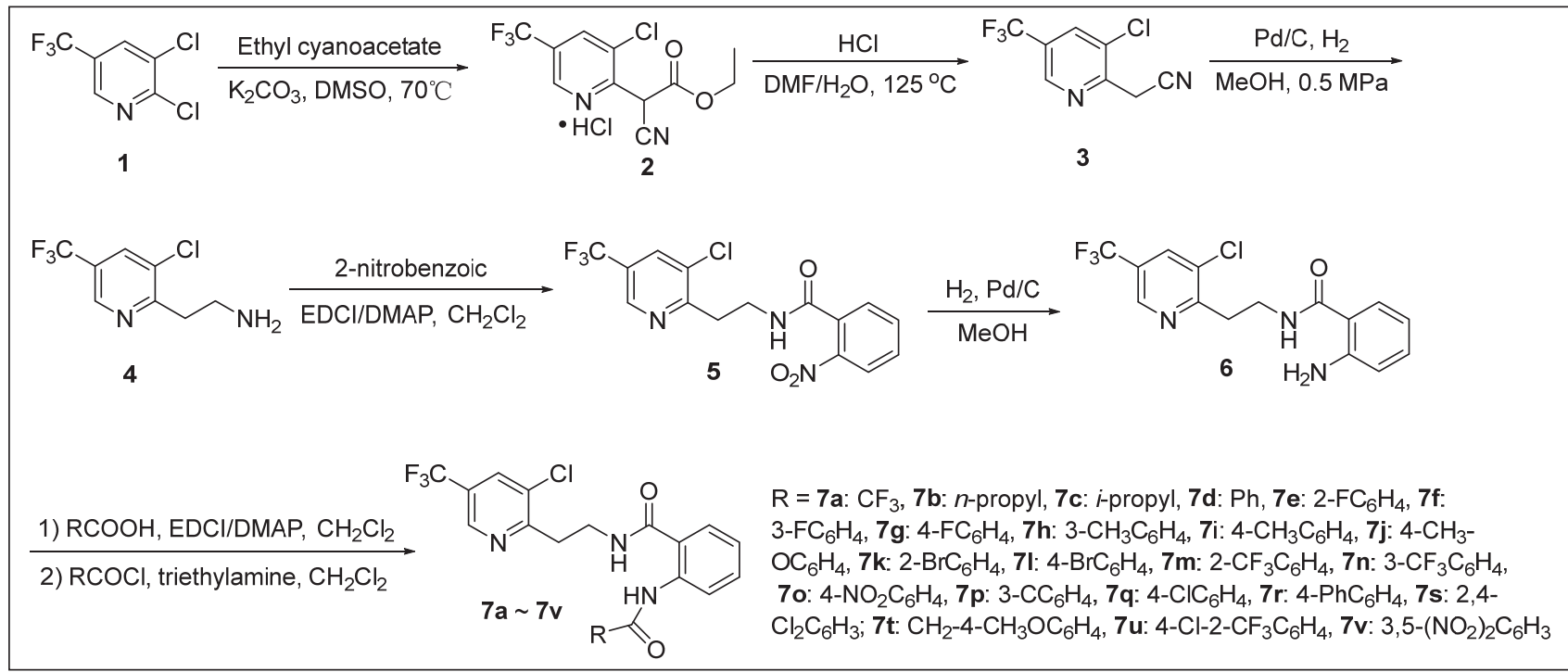

图式 1 目标化合物的合成

Scheme 1 Synthesis route of the target compounds $7 \mathbf{a} \sim 7 \mathrm{v}$

节 $\mathrm{pH}$ 为 9 , 用水稀释反应液后用乙酸乙酯萃取, 经柱层 析分离得到化合物 3.

将化合物 3 溶解在甲醇中, 加入溴代四丁基铵 (TBAB)、浓硫酸、 $10 \% \mathrm{Pd} / \mathrm{C}$, 在 $0.5 \mathrm{MPa}$ 下反应 $2 \mathrm{~h}$, 经 TLC 检测反应完成, 过滤浓缩得到粗产物 4. 与文献相 比, 粗产物 $\mathbf{4}$ 可以不经过柱层析提纯, 直接经过甲醇/无 水乙醚体系进行重结晶, 实验室中一次可以制备 $50 \mathrm{~g}$ 级 的化合物 4 , 大大节约了时间.

将化合物 4 溶解在无水二氯甲烷中, 加入 1-乙基(3-二甲基氨基丙基)碳酰二亚胺盐酸盐(EDCI)、4-二甲 氨基吡啶(DMAP) 以及邻硝基苯甲酸进行缩合反应得到 中间体 $\mathbf{5}$, 中间体 $\mathbf{5}$ 可以不经过分离提纯直接进行硝基 的还原得到 6 . 化合物 $7 \mathrm{a} \sim 7 \mathrm{c}$ 是中间体 6 与相应取代酰 氯反应制得, 其余目标化合物均为中间体 $\mathbf{6}$ 与相应取代 羧酸在 EDCI/DMAP 缩合体系下进行酰胺化制得, 该法 所得双酰胺产物易于提纯, 部分化合物用甲醇重结晶即 可.

\section{2 波谱分析}

以化合物 7a 为例, 分子结构式为 $\mathrm{C}_{17} \mathrm{H}_{12} \mathrm{ClF}_{6} \mathrm{~N}_{3} \mathrm{O}_{2}$, 对化合物的 ${ }^{1} \mathrm{H}$ NMR、 ${ }^{13} \mathrm{C}$ NMR 数据进行分析. 化学位 移 $\delta 12.89$ 的单峰归属为三氟甲基附近 $\mathrm{CONH}$ 的活泼氢, 化学位移 $\delta 8.86$ 的单峰归属为吡定环的氢, 化学位移 $\delta$ $8.60 \sim 8.53$ 的多重峰归属为吡啶环的氢, $\delta 7.61$ 的单峰归 属为剩余酰胺键的活泼氢, $\delta 4.00(\mathrm{dd}, J=11.8,5.9 \mathrm{~Hz})$ 归 属为临近吡啶环的亚甲基, $\delta 3.34(\mathrm{t}, J=5.8 \mathrm{~Hz})$ 的三重峰 则归属为另一个亚甲基. 通过 ${ }^{13} \mathrm{C} \mathrm{NMR}$ 可知, $\delta 36.7$ 与 $\delta$ 33.6 为两个亚甲基的碳. $\delta 167.9$ 与 160.5 则归属为两个 酰胺键的羰基碳, $\delta$ 125.6、124.1、120.5、117.4 归属为
三氟甲基中碳的信号峰.

\section{3 杀线虫活性测定}

目标化合物 7a $\sim 7 \mathrm{v}$ 的杀线虫活性见表 1, 以氟吡菌 酰胺为阳性对照. 在 $200 \mathrm{mg} / \mathrm{L}$ 浓度下测定了化合物的 杀线虫活性. 化合物 7f、7p 和 7s (表 1) 表现出良好的杀 线虫活性，抑制率分别为 $87 \%$ 、76\%和 $81 \%$. 当吸电子 基团如卤原子取代苯环时，化合物 7f (3-F)、7g (4-F)、

$7 \mathbf{p}(3-\mathrm{Cl}) 、 7 \mathbf{s}\left(2,4-\mathrm{Cl}_{2}\right)$ 均表现出 $70 \%$ 以上的致死活性，其 中，位于间位的氟(7f，3-F)表现出良好的抑制活性，说 明间位有利于生物活性的提高. 例如, 化合物 7p (3-Cl) 和 7q (4-Cl) 的对线虫的致死率分别为 76\%和 $47 \%$.

\section{4 抑菌活性测定}

目标化合物 $7 \mathbf{a} \sim 7 \mathrm{v}$ 对苹果腐烂病原菌 (Cytospora $s p$.)、苹果轮纹病原菌(Botryosphaeria berengeriana)、番 茄灰霉病原菌 (Botrytis cinerea Pers)、油菜菌核病原菌 (Sclerotinia sclerotiorum) 在浓度 $50 \mathrm{mg} / \mathrm{L}$ 时的抑制菌丝 生长速率结果如表 2 所示. 由表 2 可知, 在 $50 \mathrm{mg} / \mathrm{L}$ 浓 度下, 大多数化合物都有显著的抑菌活性, 其中对油菜 菌核病原菌均具有显著的抑菌活性, 大于 $80 \%$. 化合物 7d、7f、7h、7i、7n 对苹果腐烂病原菌的抑制活性超过 了 $80 \%$, 但仍低于对照药剂氟吡菌酰胺, 其中化合物 $7 \mathbf{f}$ 的抑制活性达到 $92.5 \%$, 接近阳性对照. 值得一提的是, 化合物 7d、7f、7i 对四种病原菌均表现出较为优异的抑 制活性，对普篮活性较高的化合物进行了 $\mathrm{EC}_{50}$ 的测定， 结果如表 3 所示. 化合物 7d、7f、7i 对苹果腐烂病原菌 的 $\mathrm{EC}_{50}$ 分别为 $7.39 、 6.12 、 16.4 \mathrm{mg} / \mathrm{L}$, 对苹果腐烂菌有 较强的抑制活性, 并且三种化合物的抑制活性均高于阳 性对照氟吡菌酰胺 $(35.5 \mathrm{mg} / \mathrm{L})$. 
表 1 目标化合物对秀丽隐杆线虫的杀线虫活性 $(200 \mathrm{mg} / \mathrm{L})$

Table 1 Inhibition rate of compounds $7 \mathbf{a} \sim 7 \mathrm{v}$ against Caenorhabditis elegans in test tubes $(200 \mathrm{mg} / \mathrm{L})$

\begin{tabular}{|c|c|c|}
\hline Compd. & $\mathrm{R}$ & Mortality rate $/ \%$ \\
\hline $7 \mathbf{a}$ & $\mathrm{CF}_{3}$ & 20 \\
\hline $7 b$ & $n$-Propyl & 45 \\
\hline $7 \mathrm{c}$ & $i$-Propyl & 45 \\
\hline $7 d$ & $\mathrm{Ph}$ & 50 \\
\hline $7 e$ & $2-\mathrm{FC}_{6} \mathrm{H}_{4}$ & 55 \\
\hline $7 f$ & $3-\mathrm{FC}_{6} \mathrm{H}_{4}$ & 87 \\
\hline $7 \mathrm{~g}$ & $4-\mathrm{FC}_{6} \mathrm{H}_{4}$ & 70 \\
\hline $7 \mathrm{~h}$ & $3-\mathrm{CH}_{3} \mathrm{C}_{6} \mathrm{H}_{4}$ & 60 \\
\hline $7 \mathbf{i}$ & $4-\mathrm{CH}_{3} \mathrm{C}_{6} \mathrm{H}_{4}$ & 0 \\
\hline $7 \mathbf{j}$ & $4-\mathrm{CH}_{3} \mathrm{OC}_{6} \mathrm{H}_{4}$ & 0 \\
\hline $7 \mathbf{k}$ & $2-\mathrm{BrC}_{6} \mathrm{H}_{4}$ & 30 \\
\hline 71 & $4-\mathrm{BrC}_{6} \mathrm{H}_{4}$ & 20 \\
\hline $7 \mathrm{~m}$ & $2-\mathrm{CF}_{3} \mathrm{C}_{6} \mathrm{H}_{4}$ & 55 \\
\hline $7 n$ & $3-\mathrm{CF}_{3} \mathrm{C}_{6} \mathrm{H}_{4}$ & 10 \\
\hline 70 & $4-\mathrm{NO}_{2} \mathrm{C}_{6} \mathrm{H}_{4}$ & 15 \\
\hline $7 p$ & $3-\mathrm{ClC}_{6} \mathrm{H}_{4}$ & 76 \\
\hline $7 q$ & $4-\mathrm{ClC}_{6} \mathrm{H}_{4}$ & 47 \\
\hline $7 r$ & 4-PhC $6 \mathrm{H}_{4}$ & 0 \\
\hline $7 s$ & $2,4-\mathrm{Cl}_{2} \mathrm{C}_{6} \mathrm{H}_{4}$ & 81 \\
\hline $7 t$ & $\mathrm{CH}_{2}-\left(4-\mathrm{OCH}_{3}\right) \mathrm{C}_{6} \mathrm{H}_{4}$ & 70 \\
\hline $7 u$ & $4-\mathrm{Cl}-2-\mathrm{CF}_{3} \mathrm{C}_{6} \mathrm{H}_{4}$ & 50 \\
\hline $7 v$ & $3,5-\left(\mathrm{NO}_{2}\right)_{2} \mathrm{C}_{6} \mathrm{H}_{4}$ & 66 \\
\hline Fluopyram & & 100 \\
\hline
\end{tabular}

通过对活性的分析得知, 当苯环上引入给电子基团 时会降低杀菌活性, 如当引入烷氧基后对苹果腐烂病原 菌的活性会有所降低 $(\mathbf{7} \mathbf{j}<\mathbf{7 d})$. 如果苯环引入过大的疏 水基团时会使得活性下降(7r, 22.8\%). 当苯环上引入氟 原子后会对活性有所改善(7f $>7 \mathbf{7 d}$ ), 推测可能是由于氟 原子的电负性使得苯环电子云密度降低导致活性有所 提高. 并且间位取代相对于对位和邻位取代会对活性有 较大提高 $(7 \mathrm{f}>7 \mathrm{e}>7 \mathrm{~g})$. 这些结果表明目标化合物的抑 菌活性是多种因素的共同作用.

\section{5 酶活性测定}

为了确定目标化合物对琥珀酸脱氢酶的抑制活性, 在 $100 \mu \mathrm{mol} \cdot \mathrm{L}^{-1}$ 的浓度下对化合物 $7 \mathbf{a} \sim 7 \mathbf{v}$ 对苹果腐烂 病原菌的琥珀酸脱氢酶的抑制活性进行了测定, 结果如 图 3 所示. 从图 3 可以看出, 目标化合物均表现出中等 的酶抑制活性. 其中化合物 7f 的抑制率达到 $90.1 \%$, 接 近于对照药剂氟吡菌酰胺. 并且通过对比酶活性与抑菌 活性发现, 化合物的酶抑制活性趋势与抑制苹果腐烂病
表 2 目标化合物对 4 种植物病原菌的抑制率 $(50 \mathrm{mg} / \mathrm{L})$ Table 2 Antifungal activities of the target compounds (50 $\mathrm{mg} / \mathrm{L}$ )

\begin{tabular}{ccccc}
\hline & \multicolumn{4}{c}{ Inhibition rate (control effects)/\% } \\
\cline { 2 - 5 } Compd. & $\begin{array}{c}\text { Cytospora } \\
\text { sp. }\end{array}$ & $\begin{array}{c}\text { Botryosphaeria } \\
\text { berengeriana }\end{array}$ & $\begin{array}{c}\text { Botrytis } \\
\text { cinerea } \\
\text { Pers }\end{array}$ & $\begin{array}{c}\text { Sclerotinia } \\
\text { sclerotiorum }\end{array}$ \\
\hline $\mathbf{7 a}$ & 73.3 & 37.6 & 13.9 & 81.3 \\
$\mathbf{7 b}$ & 73.3 & 16.1 & 36.1 & 72.9 \\
$\mathbf{7 c}$ & 55.0 & 30.6 & 34.7 & 72.9 \\
$\mathbf{7 d}$ & 89.4 & 55.9 & 41.7 & 88.2 \\
$\mathbf{7 e}$ & 43.9 & 14.0 & 0 & 15.3 \\
$\mathbf{7 f}$ & 92.5 & 53.8 & 55.6 & 88.9 \\
$\mathbf{7 g}$ & 23.9 & 22.0 & 5.6 & 5.6 \\
$\mathbf{7 h}$ & 84.4 & 2.7 & 4.9 & 69.4 \\
$\mathbf{7 i}$ & 84.4 & 33.9 & 45.1 & 88.9 \\
$\mathbf{7 j}$ & 79.4 & 32.8 & 55.6 & 90.3 \\
$\mathbf{7 k}$ & 19.4 & 0 & 0 & 0 \\
$\mathbf{7 1}$ & 62.2 & 10.8 & 10.0 & 85.4 \\
$\mathbf{7 m}$ & 40.6 & 37.6 & 22.2 & 87.5 \\
$\mathbf{7 n}$ & 84.4 & 2.7 & 4.9 & 69.4 \\
$\mathbf{7 0}$ & 21.1 & 0 & 13.9 & 82.6 \\
$\mathbf{7 p}$ & 65.0 & 0 & 54.5 & 32.5 \\
$\mathbf{7 q}$ & 36.7 & 19.9 & 36.8 & 77.8 \\
$\mathbf{7 r}$ & 22.8 & 8.1 & 0 & 12.5 \\
$\mathbf{7} \mathbf{7}$ & 60.6 & 5.9 & 0 & 81.9 \\
$\mathbf{7 t}$ & 61.1 & 34.9 & 0 & 14.6 \\
$\mathbf{7 u}$ & 28.3 & 7.0 & 18.1 & 2.8 \\
$\mathbf{7 v}$ & 34.4 & 15.1 & 0 & 80.6 \\
Fluopyram & 95.0 & 90.0 & 91.0 & 92.0 \\
\hline & & & & \\
\hline
\end{tabular}

表 3 化合物 7d、7f、7i 以及氟吡菌酰胺对苹果腐烂病原菌的 $\mathrm{EC}_{50}$

Table 3 EC50 values of $\mathbf{7 d}, \mathbf{7 f}, \mathbf{7 i}$, and fluopyram against $C y t o-$ spora sp.

\begin{tabular}{cclc}
\hline Compd. & $\mathrm{EC}_{50} /\left(\mathrm{mol} \cdot \mathrm{L}^{-1}\right)$ & \multicolumn{1}{c}{ Equation } & $\mathrm{R}^{2}$ \\
\hline $\mathbf{7 d}$ & 7.39 & $Y=1.4509 x+3.74$ & 0.98 \\
$\mathbf{7 f}$ & 6.12 & $Y=1.971 x+3.3379$ & 0.99 \\
$\mathbf{7 i}$ & 16.4 & $Y=0.8564 x+3.9593$ & 0.99 \\
Fluopyram & 35.5 & $Y=1.8794 x+2.0858$ & 0.99 \\
\hline
\end{tabular}

原菌活性趋势相近，表明所制备化合物可以通过抑制病 原真菌的呼吸作用从而达到杀菌作用.

\section{6 分子对接}

为了探究目标化合物与靶标酶之间的结合方式, 对 化合物 7f 和 7k 以及氟吡菌酰胺进行了分子对接. 从蛋 白质结构数据库种选取 SDH 晶体(4ysz)作为蛋白受体模 板, 经过去除水分子、加氢等处理后进行分子对接, 结 果如图 4 所示. 化合物 7f 与酶的结合方式与氟吡菌酰胺 的结合方式类似，化合物 $7 \mathbf{f}$ 的吡啶环深入到活性口袋 的疏水区，与 $\operatorname{Trp} 67$ 形成 $\pi-\pi$ 相互作用，酰胺键的氧原子 与 Pro211 形成氢键相互作用. 与氟吡菌酰胺不同的是, 氟吡菌酰胺的氢键长度为 $0.2 \mathrm{~nm}$, 而 $7 \mathbf{f}$ 形成的氢键长度 


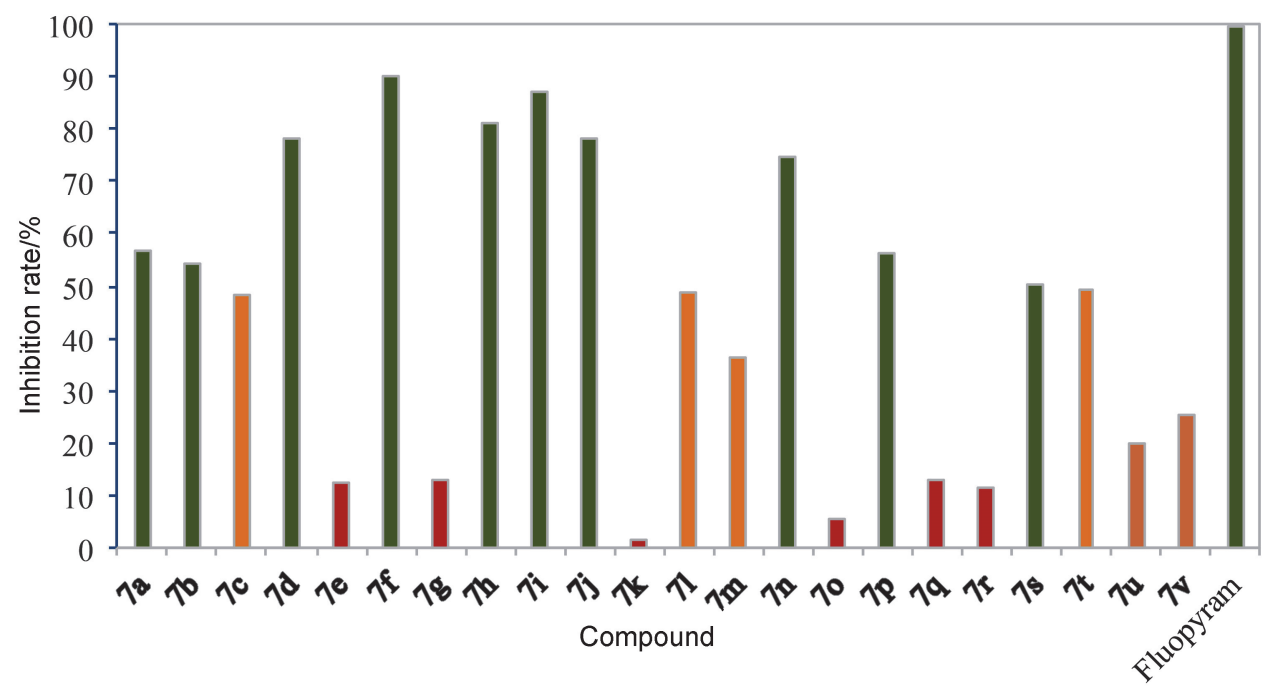

图 3 目标化合物对苹果腐烂病原菌的 $\mathrm{SDH}$ 酶活性 $\left(100 \mu \mathrm{mol} \cdot \mathrm{L}^{-1}\right)$

Figure 3 Inhibition rate of title compounds $7 \mathbf{a} \sim 7 \mathbf{v}$ to Cytospora sp $\operatorname{SDH}\left(100 \mu \mathrm{mol} \cdot \mathrm{L}^{-1}\right)$

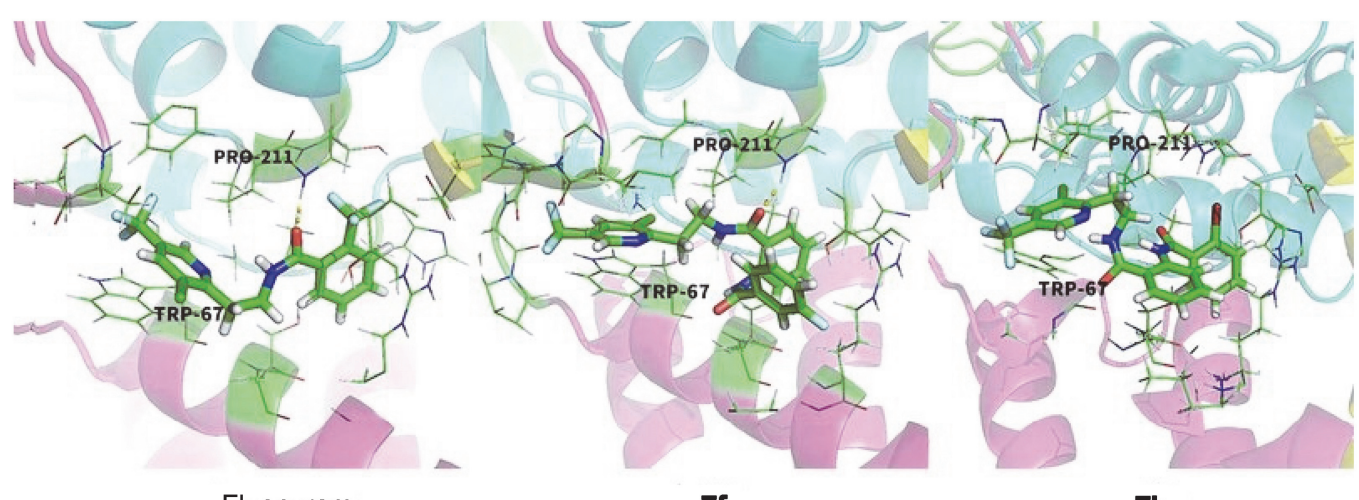

Fluopyram

$7 f$

7k

图 4 目标化合物与 SDH 酶分子对接图

Figure 4 Docking modes of Title compounds with SDH

为 $0.18 \mathrm{~nm}$, 由于双酰胺结构的引入导致酰胺键发生了 一定程度的翻转, 从而拉近了与关键氨基酸的距离, 从 而增强了化合物与靶酶的相互作用力. 反观 $7 \mathbf{k}$, 由于邻 位溴原子的引入导致分子的构象发生了较大程度的翻 转, 从而导致吡啶环不能在疏水区与 $\operatorname{Trp} 67$ 形成 $\pi-\pi$ 相 互作用, 并且翻转导致酰胺键的氧原子远离 Pro211, 从 而不能形成氢键相互作用, 这也是 $7 \mathbf{k}$ 的杀菌活性差的 原因, 与实验结果一致.

分子对接表明化合物 7f 与 $\mathrm{SDH}$ 会形成较强的相互 作用力, 与抑菌实验以及酶抑制活性实验结果一致. 综 合酶活性和分子对接结果可知, SDH 酶可能是所合成目 标化合物的潜在靶标.

\section{2 结论}

基于亚结构拼接原理, 以氟吡菌酰胺为先导化合 物, 设计并合成了 22 个未见文献报道的新颖双酰胺衍 生物, 对目标化合物进行了抑菌活性、杀线虫活性以及
酶活性的测定. 实验结果表明, 在浓度为 $200 \mathrm{mg} / \mathrm{L}$ 时, 化合物 7f 表现出中等的杀线虫活性，死亡率为 $87 \%$. 在 $50 \mathrm{mg} / \mathrm{L}$ 浓度下, 22 个化合物对四种病原菌均表现出一 定的杀菌活性，其中 $7 \mathbf{f}$ 对苹果腐烂病原菌的抑制率为 $92.5 \%, \mathrm{EC}_{50}$ 为 $6.12 \mathrm{mg} / \mathrm{L}$, 超过阳性对照氟吡菌酰胺. 目标化合物对苹果腐烂病原菌 SDH 酶活性与抑菌活性 趋势一致. 分子对接研究揭示 SDH 可能是新合成双酰 胺衍生物的潜在靶标.

\section{3 实验部分}

\section{1 仪器与试剂}

核磁共振氢谱和碳谱通过 BRUKER Avance 300 $\mathrm{MHz}$ 核磁共振仪(TMS 为内标)测定，高分辨质谱通过 Bruker Daltonics Bio-TOF-Q III 型质谱仪(ESIMS)测定. 实验中所用试剂为国产市售分析纯试剂. 中间体 2、3、 4 参考文献[19]方法制备. 


\section{2 目标化合物的合成}

\section{2 .1 化合物 $\mathbf{5}$ 的合成}

在 $100 \mathrm{~mL}$ 的圆底烧瓶中加入化合物 $42.24 \mathrm{~g}(10$ $\mathrm{mmol})$, 用 $15 \mathrm{~mL}$ 无水二氯甲烷溶解, 加入 EDCI $2.9 \mathrm{~g}$

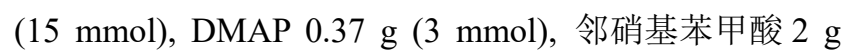
(12 mmol), 溶解完全后, 在氮气保护下室温反应 $6 \mathrm{~h}$, 薄层色谱 $[$ TLC, $V$ (乙酸乙酯) $: V$ (甲醇) $: V($ 水 $)=8: 1$ ： 1]检测, 反应完全. 停止反应, 在反应液中加入 $10 \mathrm{~mL}$ 二氯甲烷, 依次用稀盐酸, 饱和碳酸氢钠溶液, 饱和食 盐水洗涤, 二氯甲烷萃取 $(5 \mathrm{~mL} \times 2)$, 有机相用无水 $\mathrm{Na}_{2} \mathrm{SO}_{4}$ 干燥, 过滤, 旋转蒸发仪蒸出多余溶剂, 剩余物 经过柱层析 $[V$ (石油醚) $: V($ 乙酸乙酯 $)=5: 1]$ 分离得到 乳白色固体 N-(2-(3-氯-5-(三氟甲基)吡啶-2-基)乙基)-2硝基苯甲酰胺 (5) (3.13 g, 收率 84\%). m.p.132 134 ${ }^{\circ} \mathrm{C}$; ${ }^{1} \mathrm{H}$ NMR $\left(300 \mathrm{MHz}, \mathrm{CDCl}_{3}\right) \delta: 8.66(\mathrm{~d}, J=1.0 \mathrm{~Hz}, 1 \mathrm{H}$, Py-H), 8.02 (dd, $J=8.1,1.0 \mathrm{~Hz}, 1 \mathrm{H}, \mathrm{Py}-\mathrm{H}), 7.93$ (d, $J=$ $1.6 \mathrm{~Hz}, 1 \mathrm{H}, \mathrm{Ph}-\mathrm{H}), 7.67$ (td, $J=7.5,1.3 \mathrm{~Hz}, 1 \mathrm{H}, \mathrm{Ph}-\mathrm{H})$, 7.53 (s, 2H, Ph-H), 6.79 (s, 1H, NH), 4.01 (d, $J=5.8 \mathrm{~Hz}$, $\left.2 \mathrm{H}, \mathrm{CH}_{2}\right), 3.36$ (s, $\left.2 \mathrm{H}, \mathrm{CH}_{2}\right) ;{ }^{13} \mathrm{C} \mathrm{NMR}\left(126 \mathrm{MHz}, \mathrm{CDCl}_{3}\right)$ $\delta: 165.4,159.9,145.5,142.6,132.9,132.7,132.0,131.0$, 129.4, 127.7, 125.0, 124.8, 123.5, 122.8, 120.6, 76.3, 76.0, 75.8, 36.0, 32.9. HRMS (ESI) calcd for $\mathrm{C}_{15} \mathrm{H}_{11} \mathrm{ClF}_{3} \mathrm{~N}_{3} \mathrm{O}_{3}$ $[\mathrm{M}+\mathrm{H}]^{+}$373.0441, found 373.0452 .

\section{2 .2 化合物 6 的合成}

在 $50 \mathrm{~mL}$ 圆底烧瓶中加入化合物 $5(3.73 \mathrm{~g}, 10$ $\mathrm{mmol})$ 和 $10 \mathrm{~mL}$ 甲醇, $10 \% \mathrm{Pd} / \mathrm{C}(0.3 \mathrm{~g})$ 溶解在 $5 \mathrm{~mL}$ 甲醇 中加入到反应体系中, 通过三通连接储气球, 将反应体 系抽真空, 然后充入氮气, 重复 2 次后, 充入氢气. 在室 温下反应 $2 \mathrm{~h}$, 薄层色谱 $[V$ (石油醚)： $V$ (乙酸乙酯 $)=1$ : 1]检测, 反应完全后停止反应, 将反应体系过滤, 回收 $\mathrm{Pd} / \mathrm{C}$. 反应体系真空浓缩溶剂后得到黄色固体, 甲醇重 结晶得到 2-氨基- $N$-(2-(3-氯-5-(三氟甲基)吡啶-2-基)乙 基)苯甲酰胺(6) $(3.1 \mathrm{~g}, 90 \%)$. m.p. $108 \sim 110{ }^{\circ} \mathrm{C} ;{ }^{1} \mathrm{H}$ NMR (300 MHz, $\left.\mathrm{CDCl}_{3}\right) \delta: 8.84$ (s, 1H, Py-H), $7.88(\mathrm{dd}$, $J=8.1,2.3 \mathrm{~Hz}, 1 \mathrm{H}, \mathrm{Py}-\mathrm{H}), 7.33$ (dd, $J=13.0,4.8 \mathrm{~Hz}, 2 \mathrm{H}$ ), 7.21 (ddd, $J=8.3,7.2,1.5 \mathrm{~Hz}, 1 \mathrm{H}), 7.12 \sim 7.04(\mathrm{~m}, 1 \mathrm{H}$, Ph-H), 6.67 (ddd, $J=8.9,6.1,2.0 \mathrm{~Hz}, 2 \mathrm{H}, \mathrm{Ph}-\mathrm{H}$ ), 5.56 (s, $2 \mathrm{H}, \mathrm{NH}), 3.87$ (dd, $\left.J=12.3,5.8 \mathrm{~Hz}, 2 \mathrm{H}, \mathrm{CH}_{2}\right), 3.19$ (t, $J=$ $\left.6.2 \mathrm{~Hz}, 2 \mathrm{H}, \mathrm{CH}_{2}\right) ;{ }^{13} \mathrm{C} \mathrm{NMR}\left(126 \mathrm{MHz}, \mathrm{CDCl}_{3}\right) \delta: 168.2$, $160.1,142.7,132.9,132.8,131.2,130.9,126.1,125.0$, 124.8, 122.9, 122.4, 120.6, 116.5, 115.9, 115.3, 35.7, 33.5. HRMS (ESI) calcd for $\mathrm{C}_{15} \mathrm{H}_{13} \mathrm{ClF}_{3} \mathrm{~N}_{3} \mathrm{O}[\mathrm{M}+\mathrm{H}]^{+}$ 343.0699, found 343.0671.

3.2.3 化合物 $7 \mathbf{a} \sim 7 \mathbf{c}$ 的合成

在 $50 \mathrm{~mL}$ 圆底烧瓶中加入化合物 $60.8 \mathrm{~g} \mathrm{(2.33}$ $\mathrm{mmol}$ )和三乙胺 $0.35 \mathrm{~g}(3.5 \mathrm{mmol}), 10 \mathrm{~mL}$ 无水二氯甲烷 作为溶剂, 在冰浴下滴加三氟乙酰氯 $0.34 \mathrm{~g}(2.56 \mathrm{mmol})$ 的无水二氯甲烷溶液, 滴加完毕后室温反应 $3 \mathrm{~h}$. 薄层 色谱 $[V$ (石油醚) $: V$ (乙酸乙酯 $)=1: 1]$ 检测, 反应完全. 反应体系依次用饱和碳酸氢钠溶液以及饱和食盐水洗 涤，二氯甲烷萃取 $(10 \mathrm{~mL} \times 2)$, 有机相用无水 $\mathrm{Na}_{2} \mathrm{SO}_{4}$ 干 燥, 过滤, 母液浓缩至干, 所得粗品经柱层析 $[V$ (石油 醚)：V(乙酸乙酯 $)=5: 1]$ 纯化得到目标化合物 $N-(2-(3-$ 氯-5-(三氟甲基)吡啶-2-基)乙基)-2-(2,2,2-三氟乙酰氨 基)苯甲酰胺 $(7 \mathbf{a}), 0.87 \mathrm{~g}$, 收率 $85 \%$. 白色固体, m.p. 134 136 ${ }^{\circ} \mathrm{C}$; ${ }^{1} \mathrm{H}$ NMR $\left(300 \mathrm{MHz}, \mathrm{CDCl}_{3}\right) \delta: 12.89$ (s, $1 \mathrm{H}, \mathrm{NH}), 8.76$ (s, $1 \mathrm{H}, \mathrm{Py}-\mathrm{H}), 8.60 \sim 8.53(\mathrm{~m}, 1 \mathrm{H}, \mathrm{Py}-\mathrm{H})$, 7.94 (d, $J=1.7 \mathrm{~Hz}, 1 \mathrm{H}, \mathrm{Ph}-\mathrm{H}), 7.61$ (s, 1H, NH), 7.57 7.42 (m, 2H, Ph-H), 7.26 7.18 (m, 1H, Ph-H), 4.00 (dd, $\left.J=11.8,5.9 \mathrm{~Hz}, 2 \mathrm{H}, \mathrm{CH}_{2}\right), 3.34\left(\mathrm{t}, J=5.8 \mathrm{~Hz}, 2 \mathrm{H}, \mathrm{CH}_{2}\right)$; ${ }^{13} \mathrm{C}$ NMR $\left(75 \mathrm{MHz}, \mathrm{CDCl}_{3}\right) \delta: 167.9,160.7,155.1,154.6$, $143.3,137.5,133.7,132.6,131.7,126.2,125.6,124.4$, $124.1,123.0,121.2,120.5,120.0,117.4,113.5,77.1,76.7$, 76.3, 36.7, 33.6. HRMS (ESI) calcd for $\mathrm{C}_{17} \mathrm{H}_{13} \mathrm{ClF}_{6} \mathrm{~N}_{3} \mathrm{O}_{2}$ $[\mathrm{M}+\mathrm{H}]^{+} 440.0595$, found 440.0588 .

化合物 $7 \mathbf{b} \sim 7 \mathbf{c}$ 的合成参照化合物 $7 \mathbf{a}$ 的合成方法.

2-丁胺基- $N$-(2-(3-氯-5-(三氟甲基)吡啶-2-基)乙基) 苯甲酰胺 (7b): 黄色油状液体, 收率 87\%. ${ }^{1} \mathrm{H}$ NMR $(300$ $\left.\mathrm{MHz}, \mathrm{CDCl}_{3}\right) \delta: 11.15(\mathrm{~s}, 1 \mathrm{H}), 8.75(\mathrm{~s}, 1 \mathrm{H}), 8.60(\mathrm{~d}, J=$ $8.1 \mathrm{~Hz}, 1 \mathrm{H}), 7.94(\mathrm{~d}, J=1.7 \mathrm{~Hz}, 1 \mathrm{H}), 7.42$ (dd, $J=7.8,2.0$ $\mathrm{Hz}, 2 \mathrm{H}), 7.35$ (s, 1H), $7.10 \sim 6.99(\mathrm{~m}, 1 \mathrm{H}), 3.97$ (d, $J=6.0$ $\mathrm{Hz}, 2 \mathrm{H}), 3.33(\mathrm{t}, J=5.9 \mathrm{~Hz}, 2 \mathrm{H}), 2.51 \sim 2.28(\mathrm{~m}, 2 \mathrm{H}), 1.77$ (dd, $J=15.0,7.5 \mathrm{~Hz}, 2 \mathrm{H}), 1.01(\mathrm{t}, J=7.4 \mathrm{~Hz}, 3 \mathrm{H}) ;{ }^{13} \mathrm{C}$ NMR $\left(75 \mathrm{MHz}, \mathrm{CDCl}_{3}\right) \delta: 171.7,168.6,160.5,143.4$, 139.4, 133.6, 132.2, 131.6, 126.1, 125.7, 124.1, 122.2, $121.1,120.5,119.8,77.1,76.7,76.3,40.1,36.6,33.8,18.7$, 13.4. HRMS (ESI) calcd for $\mathrm{C}_{19} \mathrm{H}_{20} \mathrm{ClF}_{3} \mathrm{~N}_{3} \mathrm{O}_{2}[\mathrm{M}+\mathrm{H}]^{+}$ 414.1191, found 414.1191.

$N$-(2-(3-氯-5-(三氟甲基)吡啶-2-基)乙基)-2-异丁酰 胺苯甲酰胺 $(7 \mathrm{c})$ : 黄色油状液体, 收率 $83 \% .{ }^{1} \mathrm{H}$ NMR $\left(300 \mathrm{MHz}, \mathrm{CDCl}_{3}\right) \delta: 11.22(\mathrm{~s}, 1 \mathrm{H}), 8.73(\mathrm{~d}, J=1.0 \mathrm{~Hz}$, $1 \mathrm{H}), 8.56(\mathrm{~d}, J=7.9 \mathrm{~Hz}, 1 \mathrm{H}), 7.90(\mathrm{~d}, J=1.8 \mathrm{~Hz}, 1 \mathrm{H}), 7.39$ (dd, $J=12.3,4.5 \mathrm{~Hz}, 3 \mathrm{H}), 7.00$ (td, $J=7.7,1.1 \mathrm{~Hz}, 1 \mathrm{H})$, 3.95 (q, $J=6.0 \mathrm{~Hz}, 2 \mathrm{H}), 3.32(\mathrm{t}, J=6.0 \mathrm{~Hz}, 2 \mathrm{H}), 2.56$ (dt, $J=13.8,6.9 \mathrm{~Hz}, 1 \mathrm{H}), 1.28 \sim 1.23(\mathrm{~m}, 6 \mathrm{H}) ;{ }^{13} \mathrm{C}$ NMR $(75$ $\left.\mathrm{MHz}, \mathrm{CDCl}_{3}\right) \delta: 175.7,168.6,160.5,143.4,143.3,139.5$, $133.6,132.0,131.5,126.2,125.6,124.1,122.1,121.0$, $120.5,119.9,77.1,76.7,76.3,40.1,36.9,33.9,19.1$. HRMS (ESI) calcd for $\mathrm{C}_{19} \mathrm{H}_{20} \mathrm{ClF}_{3} \mathrm{~N}_{3} \mathrm{O}_{2} \quad[\mathrm{M}+\mathrm{H}]^{+}$ 414.1191, found 414.1191. 


\section{2 .4 化合物 $7 \mathbf{d} \sim 7 \mathrm{v}$ 的合成}

在 $50 \mathrm{~mL}$ 的圆底烧瓶中加入化合物 $60.8 \mathrm{~g}(2.33$ $\mathrm{mmol})$, 用 $10 \mathrm{~mL}$ 无水二氯甲烷溶解, 加入 EDCI $0.67 \mathrm{~g}$ (3.5 mmol), DMAP $0.03 \mathrm{~g}(0.23 \mathrm{mmol})$, 苯甲酸 $0.34 \mathrm{~g}$ $(2.8 \mathrm{mmol})$, 溶解完全后, 在氮气保护下室温反应 $6 \mathrm{~h}$, 薄层色谱 $[$ TLC, $V$ (石油醚) $: V$ (乙酸乙酯 $)=1 ： 1]$ 检测, 反应完全. 停止反应, 在反应液中加入 $10 \mathrm{~mL}$ 二氯甲烷, 依次用稀盐酸、饱和碳酸氢钠溶液、饱和食盐水洗涤, 二 氯甲烷萃取 $(5 \mathrm{~mL} \times 2)$, 有机相用无水 $\mathrm{Na}_{2} \mathrm{SO}_{4}$ 干燥, 过 滤, 旋转蒸发仪蒸出多余溶剂, 剩余物经过柱层析 $[V($ 石 油醚) $: V$ (乙酸乙酯 $)=5 ： 1$ ] 分离得到白色固体 2-苯甲 酰胺- $N$-(2-(3-氯-5-(三氟甲基)吡啶-2-基)乙基)苯甲酰胺 (7d) 0.9 g, 收率 86\%. m.p. 136 $137{ }^{\circ} \mathrm{C} ;{ }^{1} \mathrm{H}$ NMR (300 $\left.\mathrm{MHz}, \mathrm{CDCl}_{3}\right) \delta: 12.22(\mathrm{~s}, 1 \mathrm{H}), 8.81 \sim 8.72(\mathrm{~m}, 2 \mathrm{H}), 8.04$ $(\mathrm{dd}, J=7.9,1.8 \mathrm{~Hz}, 2 \mathrm{H}), 7.91(\mathrm{~d}, J=1.6 \mathrm{~Hz}, 1 \mathrm{H}), 7.58 \sim$ $7.44(\mathrm{~m}, 6 \mathrm{H}), 7.11 \sim 7.02(\mathrm{~m}, 1 \mathrm{H}), 4.00(\mathrm{~d}, J=6.0 \mathrm{~Hz}, 2 \mathrm{H})$, $3.35(\mathrm{~d}, J=6.0 \mathrm{~Hz}, 2 \mathrm{H}) ;{ }^{13} \mathrm{C} \mathrm{NMR}\left(75 \mathrm{MHz}, \mathrm{CDCl}_{3}\right) \delta$ : $168.8,165.2,160.5,143.4,139.7,134.5,133.7,133.6$, $132.3,131.6,131.5,128.4,127.0,126.3,126.0,125.5$, 124.1, 122.2, 121.2, 120.5, 110.1, 77.1, 76.7, 76.3, 36.6, 33.8. HRMS (ESI) calcd for $\mathrm{C}_{22} \mathrm{H}_{18} \mathrm{ClF}_{3} \mathrm{~N}_{3} \mathrm{O}_{2}[\mathrm{M}+\mathrm{H}]^{+}$ 448.1041, found 448.1041.

化合物 $7 \mathrm{e} \sim 7 \mathrm{v}$ 的合成参照化合物 $7 \mathrm{~d}$ 的合成方法.

$N$-(2-(3-氯-5-(三氟甲基)吡啶-2-基)乙基)-2-(2-氟苯 甲酰胺基)苯甲酰胺(7e): 白色固体, 收率 $86 \%$. m.p. 146 $148{ }^{\circ} \mathrm{C} ;{ }^{1} \mathrm{H}$ NMR (300 MHz, $\left.\mathrm{CDCl}_{3}\right) \delta: 11.81$ (d, $J=7.2 \mathrm{~Hz}, 1 \mathrm{H}), 8.80$ (s, 1H), 8.67 (d, $J=8.3 \mathrm{~Hz}, 1 \mathrm{H}), 8.00$ (td, $J=7.7,1.8 \mathrm{~Hz}, 1 \mathrm{H}), 7.82(\mathrm{dd}, J=8.2,2.1 \mathrm{~Hz}, 1 \mathrm{H})$, 7.68 (s, 1H), 7.46 (ddd, $J=9.7,4.4,1.6 \mathrm{~Hz}, 3 \mathrm{H}), 7.32$ (d, $J=8.1 \mathrm{~Hz}, 1 \mathrm{H}), 7.26 \sim 7.19(\mathrm{~m}, 1 \mathrm{H}), 7.16(\mathrm{dd}, J=6.7,4.6$ $\mathrm{Hz}, 1 \mathrm{H}), 7.09 \sim 7.02(\mathrm{~m}, 1 \mathrm{H}), 3.86(\mathrm{dd}, J=12.2,6.0 \mathrm{~Hz}$, 2H), 3.17 (t, $J=6.3 \mathrm{~Hz}, 2 \mathrm{H}) ;{ }^{13} \mathrm{C} \mathrm{NMR}\left(75 \mathrm{MHz}, \mathrm{CDCl}_{3}\right)$ $\delta: 168.3,162.1,160.6,158.3,143.4,138.6,133.6,133.0$, $131.9,131.6,131.1,126.3,125.9,125.5,124.2,123.0$, $122.5,122.0,121.7,120.5,116.3,116.0,77.13,76.70$, 76.28, 36.6, 33.8. HRMS (ESI) calcd for $\mathrm{C}_{22} \mathrm{H}_{17} \mathrm{ClF}_{4} \mathrm{~N}_{3} \mathrm{O}_{2}$ $[\mathrm{M}+\mathrm{H}]^{+}$466.0940, found 466.0942.

$N$-(2-(3-氯-5-(三氟甲基)吡啶-2-基)乙基)-2-(3-氟苯 甲酰胺基)苯甲酰胺(7f): 白色固体, 收率 84\%. m.p. $139 \sim 141{ }^{\circ} \mathrm{C} ;{ }^{1} \mathrm{H}$ NMR $\left(300 \mathrm{MHz}, \mathrm{CDCl}_{3}\right) \delta: 12.40$ (s, $1 \mathrm{H}), 8.85(\mathrm{~d}, J=4.5 \mathrm{~Hz}, 1 \mathrm{H}), 8.77(\mathrm{dd}, J=17.5,8.1 \mathrm{~Hz}$, $1 \mathrm{H}), 8.13 \sim 8.00(\mathrm{~m}, 1 \mathrm{H}), 7.93 \sim 7.85(\mathrm{~m}, 1 \mathrm{H}), 7.84 \sim 7.73$ $(\mathrm{m}, 1 \mathrm{H}), 7.59 \sim 7.44(\mathrm{~m}, 3 \mathrm{H}), 7.35(\mathrm{dd}, J=14.3,8.7 \mathrm{~Hz}$, $1 \mathrm{H}), 7.22(\mathrm{dd}, J=10.4,5.9 \mathrm{~Hz}, 1 \mathrm{H}), 7.12$ (d, $J=7.6 \mathrm{~Hz}$, $1 \mathrm{H}), 3.93(\mathrm{dd}, J=11.9,6.0 \mathrm{~Hz}, 2 \mathrm{H}), 3.21(\mathrm{dd}, J=11.8,5.8$
$\mathrm{Hz}, 2 \mathrm{H}) ;{ }^{13} \mathrm{C} \mathrm{NMR}\left(75 \mathrm{MHz}, \mathrm{CDCl}_{3}\right) \delta:{ }^{13} \mathrm{C} \mathrm{NMR}(126$ $\left.\mathrm{MHz}, \mathrm{CDCl}_{3}\right) \delta: 168.1,167.6,163.4,142.7,139.1,138.9$, $138.0,133.0,131.8,131.3,130.9,128.8,125.5,122.4$, 122.1, 121.9, 121.4, 120.5, 119.1, 114.9, 114.7, 36.0, 33.1. HRMS (ESI) calcd for $\mathrm{C}_{22} \mathrm{H}_{17} \mathrm{ClF}_{4} \mathrm{~N}_{3} \mathrm{O}_{2} \quad[\mathrm{M}+\mathrm{H}]{ }^{+}$ 466.0940, found 466.0932 .

$N$-(2-(3-氯-5-(三氟甲基)吡啶-2-基)乙基)-2-(4-氟苯 甲酰胺基)苯甲酰胺(7g): 白色固体，收率 87\%. m.p. $152 \sim 154{ }^{\circ} \mathrm{C} ;{ }^{1} \mathrm{H}$ NMR $\left(500 \mathrm{MHz}, \mathrm{CDCl}_{3}\right) \delta: 12.18(\mathrm{~s}$, $1 \mathrm{H}), 8.67(\mathrm{dd}, J=10.2,1.5 \mathrm{~Hz}, 2 \mathrm{H}), 8.02 \sim 7.93(\mathrm{~m}, 2 \mathrm{H})$, $7.83(\mathrm{~d}, J=1.7 \mathrm{~Hz}, 1 \mathrm{H}), 7.46 \sim 7.32(\mathrm{~m}, 3 \mathrm{H}), 7.13 \sim 7.06$ $(\mathrm{m}, 2 \mathrm{H}), 7.03 \sim 6.95(\mathrm{~m}, 1 \mathrm{H}), 3.91(\mathrm{dd}, J=11.9,5.9 \mathrm{~Hz}$, $2 \mathrm{H}), 3.25$ (t, $J=5.9 \mathrm{~Hz}, 2 \mathrm{H}) ;{ }^{13} \mathrm{C} \mathrm{NMR}\left(126 \mathrm{MHz} \mathrm{CDCl}_{3}\right)$ $\delta: 168.1,165.0,163.4,163.0,159.8,143.2,139.0133 .0$, $131.8,130.9,130.0,128.8,125.5,125.3,125.0,121.9$, 120.4, 119.1, 114.8, 114.7, 36.0, 33.1. HRMS (ESI) calcd for $\mathrm{C}_{22} \mathrm{H}_{17} \mathrm{ClF}_{4} \mathrm{~N}_{3} \mathrm{O}_{2}[\mathrm{M}+\mathrm{H}]^{+}$466.0940, found 466.0942 .

$N$-(2-(3-氯-5-(三氟甲基)吡啶-2-基)乙基)-2-(3-甲基 苯甲酰胺基)苯甲酰胺(7h): 白色固体，收率 84\%. m.p. $141 \sim 143{ }^{\circ} \mathrm{C} ;{ }^{1} \mathrm{H}$ NMR $\left(300 \mathrm{MHz}, \mathrm{CDCl}_{3}\right) \delta: 12.12(\mathrm{~s}$, $1 \mathrm{H}), 8.76(\mathrm{dd}, J=10.1,1.5 \mathrm{~Hz}, 2 \mathrm{H}), 7.95 \sim 7.78(\mathrm{~m}, 3 \mathrm{H})$, $7.55 \sim 7.33(\mathrm{~m}, 5 \mathrm{H}), 7.13 \sim 7.03(\mathrm{~m}, 1 \mathrm{H}), 4.00(\mathrm{dd}, J=$ 12.0, 6.0 Hz, 2H), 3.34 (t, $J=5.9 \mathrm{~Hz}, 2 \mathrm{H}), 2.46$ (s, 3H); ${ }^{13} \mathrm{C} \mathrm{NMR}\left(75 \mathrm{MHz}, \mathrm{CDCl}_{3}\right) \delta: 168.8,165.5,160.5,143.4$, $139.6,138.2,134.5,133.7,132.2,131.6,128.3,127.9$, $126.3,123.9,122.4,121.2,120.2,36.8,33.9,21.1$. HRMS (ESI) calcd for $\mathrm{C}_{23} \mathrm{H}_{20} \mathrm{ClF}_{3} \mathrm{~N}_{3} \mathrm{O}_{2}[\mathrm{M}+\mathrm{H}]^{+}$462.1191, found 462.1190 .

$N$-(2-(3-氯-5-(三氟甲基)吡啶-2-基)乙基)-2-(4-甲基 苯甲酰胺基)苯甲酰胺(7i): 白色固体，收率 $88 \%$. m.p. 138 $140{ }^{\circ} \mathrm{C} ;{ }^{1} \mathrm{H}$ NMR $\left(300 \mathrm{MHz}, \mathrm{CDCl}_{3}\right) \delta: 12.19$ (s, $1 \mathrm{H}), 8.84$ (s, 1H), 8.77 (d, $J=8.2 \mathrm{~Hz}, 1 \mathrm{H}), 7.93$ (d, $J=8.1$ $\mathrm{Hz}, 2 \mathrm{H}), 7.86$ (dd, $J=8.2,2.1 \mathrm{~Hz}, 1 \mathrm{H}), 7.76$ (s, 1H), 7.50 $(\mathrm{d}, J=7.7 \mathrm{~Hz}, 2 \mathrm{H}), 7.34$ (d, $J=10.1 \mathrm{~Hz}, 2 \mathrm{H}), 7.28$ (s, 1H), 7.05 (s, 1H), $3.90(\mathrm{dd}, J=12.1,5.9 \mathrm{~Hz}, 2 \mathrm{H}), 3.20(\mathrm{t}, J=$ $6.2 \mathrm{~Hz}, 2 \mathrm{H}), 2.43(\mathrm{~s}, 3 \mathrm{H}) ;{ }^{13} \mathrm{C} \mathrm{NMR}\left(75 \mathrm{MHz}, \mathrm{CDCl}_{3}\right) \delta$ : $168.8,165.3,163.2,145.8,142.0,139.8,133.5,132.2$, $131.8,129.1,127.1,126.3,123.0,122.4,121.1,120.0$, 38.2, 36.2, 21.1. HRMS (ESI) calcd for $\mathrm{C}_{23} \mathrm{H}_{20} \mathrm{ClF}_{3} \mathrm{~N}_{3} \mathrm{O}_{2}$ $[\mathrm{M}+\mathrm{H}]^{+}$462.1191, found 462.1183 .

$N$-(2-(3-氯-5-(三氟甲基)吡啶-2-基)乙基)-2-(4-甲氧 基苯甲酰胺基)苯甲酰胺(7j): 白色固体，收率 90\%. m.p. $121 \sim 122{ }^{\circ} \mathrm{C} ;{ }^{1} \mathrm{H}$ NMR (300 MHz, $\left.\mathrm{CDCl}_{3}\right) \delta$ : 12.18 (s, $1 \mathrm{H}), 8.87 \sim 8.81(\mathrm{~m}, 1 \mathrm{H}), 8.78(\mathrm{dd}, J=9.0,1.1 \mathrm{~Hz}, 1 \mathrm{H})$, $7.94(\mathrm{~d}, J=8.2 \mathrm{~Hz}, 2 \mathrm{H}), 7.91 \sim 7.82(\mathrm{~m}, 1 \mathrm{H}), 7.71(\mathrm{~s}, 1 \mathrm{H})$, 
7.50 (d, $J=7.7 \mathrm{~Hz}, 2 \mathrm{H}), 7.31$ (d, $J=8.4 \mathrm{~Hz}, 3 \mathrm{H}), 7.06$ (td, $J=7.7,1.2 \mathrm{~Hz}, 1 \mathrm{H}), 3.91$ (d, $J=6.5 \mathrm{~Hz}, 2 \mathrm{H}), 3.21(\mathrm{~s}, 2 \mathrm{H})$, 2.43 (s, 3H); ${ }^{13} \mathrm{C}$ NMR $\left(75 \mathrm{MHz}, \mathrm{CDCl}_{3}\right) \delta: 168.8,165.3$, $145.8,141.9,139.8,133.5,132.2,131.8,129.1,127.1$, $126.3,124.9,124.4,123.0,122.4,121.3,120.0,38.2,36.2$, 21.1. HRMS (ESI) calcd for $\mathrm{C}_{23} \mathrm{H}_{20} \mathrm{ClF}_{3} \mathrm{~N}_{3} \mathrm{O}_{3}[\mathrm{M}+\mathrm{Na}]^{+}$ 450.1400 , found 450.1406 .

2-溴- $N$-(2-((2-(3-氯-5-(三氟甲基)吡啶-2-基)乙基)氨 甲酰基)苯基)苯甲酰胺(7k): 白色固体, 收率 $85 \%$. m.p. $160 \sim 162{ }^{\circ} \mathrm{C}$; ${ }^{1} \mathrm{H}$ NMR (300 MHz, $\mathrm{CDCl}_{3}$ ) $\delta: 12.35$ (s, $1 \mathrm{H}), 8.9 \sim 8.66(\mathrm{~m}, 2 \mathrm{H}), 7.93(\mathrm{dd}, J=6.4,4.5 \mathrm{~Hz}, 3 \mathrm{H})$, $7.72 \sim 7.62(\mathrm{~m}, 2 \mathrm{H}), 7.58 \sim 7.43(\mathrm{~m}, 3 \mathrm{H}), 7.16 \sim 7.08(\mathrm{~m}$, $1 \mathrm{H}), 4.09(\mathrm{dd}, J=11.9,5.9 \mathrm{~Hz}, 2 \mathrm{H}), 3.36(\mathrm{t}, J=5.9 \mathrm{~Hz}$, $2 \mathrm{H}) ;{ }^{13} \mathrm{C}$ NMR $\left(75 \mathrm{MHz}, \mathrm{CDCl}_{3}\right) \delta: 168.4,166.0,163.2$, $145.8,139.0,138.1,134.1,133.3,132.3,131.0,128.6$, 127.3, 126.3, 123.1, 121.3, 120.5, 119.4, 38.2, 36.1. HRMS (ESI) calcd for $\mathrm{C}_{22} \mathrm{H}_{17} \mathrm{BrClF}_{3} \mathrm{~N}_{3} \mathrm{O}_{2}[\mathrm{M}+\mathrm{H}]^{+}$526.0139, found 526.0135 .

2-(4-溴苯甲酰胺)- $N$-(2-(3-氯-5-(三氟甲基)吡啶-2基)乙基)苯甲酰胺(71): 白色固体, 收率 82\%. m.p. 163 $165{ }^{\circ} \mathrm{C} ;{ }^{1} \mathrm{H}$ NMR $\left(300 \mathrm{MHz}, \mathrm{CDCl}_{3}\right) \delta: 12.33(\mathrm{~s}, 1 \mathrm{H})$, $8.85 \sim 8.66(\mathrm{~m}, 2 \mathrm{H}), 7.90(\mathrm{dd}, J=6.4,4.5 \mathrm{~Hz}, 3 \mathrm{H}), 7.70 \sim$ $7.61(\mathrm{~m}, 2 \mathrm{H}), 7.57 \sim 7.42(\mathrm{~m}, 3 \mathrm{H}), 7.14 \sim 7.04(\mathrm{~m}, 1 \mathrm{H})$, 4.00 (dd, $J=11.9,5.9 \mathrm{~Hz}, 2 \mathrm{H}), 3.34$ (t, $J=5.9 \mathrm{~Hz}, 2 \mathrm{H})$; ${ }^{13} \mathrm{C}$ NMR $\left(75 \mathrm{MHz}, \mathrm{CDCl}_{3}\right) \delta: 168.7,164.2,160.5,143.3$, $139.7,134.8,132.4,132.0,131.6,128.7,126.2,125.6$, 124.1, 122.6, 121.1, 120.5, 119.8, 36.7, 33.8. HRMS (ESI) calcd for $\mathrm{C}_{22} \mathrm{H}_{17} \mathrm{BrClF}_{3} \mathrm{~N}_{3} \mathrm{O}_{2}[\mathrm{M}+\mathrm{H}]^{+}$526.0139, found 526.0135 .

$N$-(2-(3-氯-5-(三氟甲基)吡啶-2-基)乙基)-2-(2(三氟 甲基)苯甲酰胺基)苯甲酰胺(7m): 白色固体, 收率 $89 \%$. m.p. $112 \sim 1116{ }^{\circ} \mathrm{C} ;{ }^{1} \mathrm{H}$ NMR (300 MHz, $\left.\mathrm{CDCl}_{3}\right) \delta: 11.72$ (s, 1H), $8.80(\mathrm{~s}, 1 \mathrm{H}), 8.69(\mathrm{~d}, J=8.1 \mathrm{~Hz}, 1 \mathrm{H}), 7.83(\mathrm{dd}$, $J=7.9,2.1 \mathrm{~Hz}, 2 \mathrm{H}), 7.73(\mathrm{~d}, J=7.5 \mathrm{~Hz}, 1 \mathrm{H}), 7.59$ (dd, $J=$ $18.3,6.8 \mathrm{~Hz}, 3 \mathrm{H}), 7.52 \sim 7.44(\mathrm{~m}, 2 \mathrm{H}), 7.09$ (dd, $J=7.6$, $0.9 \mathrm{~Hz}, 1 \mathrm{H}), 3.78$ (d, $J=6.2 \mathrm{~Hz}, 2 \mathrm{H}), 3.12(\mathrm{~d}, J=7.2 \mathrm{~Hz}$, $2 \mathrm{H}) ;{ }^{13} \mathrm{C}$ NMR (75 MHz, $\left.\mathrm{CDCl}_{3}\right) \delta: 168.3,165.8,163.2$, $145.8,139.1,136.1,133.5,132.3,131.8,129.7,127.8$, $127.5,127.1,126.7,125.3,124.4,123.1,121.7,120.3$, 38.1, 36.0. HRMS (ESI) calcd for $\mathrm{C}_{23} \mathrm{H}_{16} \mathrm{ClF}_{6} \mathrm{~N}_{3} \mathrm{O}_{2} \mathrm{Na}$ $[\mathrm{M}+\mathrm{Na}]^{+}$538.0727, found 538.0725.

$N$-(2-(3-氯-5-(三氟甲基)吡啶-2-基)乙基)-2-(3-(三氟 甲基)苯甲酰胺基)苯甲酰胺(7n): 白色固体, 收率 $85 \%$. m.p. $150 \sim 152{ }^{\circ} \mathrm{C} ;{ }^{1} \mathrm{H}$ NMR $\left(300 \mathrm{MHz}, \mathrm{CDCl}_{3}\right) \delta: 12.49$ (s, 1H), 8.77 (dd, $J=6.7,4.8 \mathrm{~Hz}, 2 \mathrm{H}), 8.36(\mathrm{~s}, 1 \mathrm{H}), 8.20$ (d, $J=7.8 \mathrm{~Hz}, 1 \mathrm{H}), 7.93(\mathrm{~d}, J=1.9 \mathrm{~Hz}, 1 \mathrm{H}), 7.82(\mathrm{~d}, J=$ $7.7 \mathrm{~Hz}, 1 \mathrm{H}), 7.67$ (t, $J=7.8 \mathrm{~Hz}, 1 \mathrm{H}, 7.58 \sim 7.35(\mathrm{~m}, 3 \mathrm{H})$, $7.17 \sim 7.07(\mathrm{~m}, 1 \mathrm{H}), 4.06 \sim 3.89(\mathrm{~m}, 2 \mathrm{H}), 3.35(\mathrm{t}, J=5.9$ $\mathrm{Hz}, 2 \mathrm{H}) ;{ }^{13} \mathrm{C}$ NMR $\left(75 \mathrm{MHz}, \mathrm{CDCl}_{3}\right) \delta: 168.7,163.7$, $160.5,145.9,143.4,139.5,135.5,133.66,132.5,131.6$, $131.3,130.8,129.8,129.0,128.0,126.4,125.6,125.3$, 124.6, 124.1, 122.9, 121.7, 121.2, 120.5, 119.8, 36.7, 33.8. HRMS (ESI) calcd for $\mathrm{C}_{23} \mathrm{H}_{17} \mathrm{ClF}_{6} \mathrm{~N}_{3} \mathrm{O}_{2} \quad[\mathrm{M}+\mathrm{H}]^{+}$ 516.0908 , found 516.0909 .

$\mathrm{N}$-(2-(3-氯-5-(三氟甲基)吡啶-2-基)乙基)-2-(4-硝基 苯甲酰胺)苯甲酰胺(7o): 黄色固体, 收率 90\%. m.p. $162 \sim 164{ }^{\circ} \mathrm{C} ;{ }^{1} \mathrm{H}$ NMR (300 MHz, $\left.\mathrm{CDCl}_{3}\right) \delta: 12.67$ (s, $1 \mathrm{H}), 8.78(\mathrm{~d}, J=8.5 \mathrm{~Hz}, 2 \mathrm{H}), 8.39 \sim 8.31(\mathrm{~m}, 2 \mathrm{H}), 8.24 \sim$ $8.16(\mathrm{~m}, 2 \mathrm{H}), 7.94(\mathrm{~d}, J=1.7 \mathrm{~Hz}, 1 \mathrm{H}), 7.60 \sim 7.48(\mathrm{~m}$, $3 \mathrm{H}), 7.19 \sim 7.10(\mathrm{~m}, 1 \mathrm{H}), 4.07 \sim 3.97(\mathrm{~m}, 2 \mathrm{H}), 3.35(\mathrm{t}, J=$ $5.9 \mathrm{~Hz}, 2 \mathrm{H}) ;{ }^{13} \mathrm{C} \mathrm{NMR}\left(75 \mathrm{MHz}, \mathrm{CDCl}_{3}\right) \delta: 168.6,162.9$, $160.4,149.4,143.4,140.1,139.5,133.7,132.6,131.6$, 128.2, 126.2, 123.6, 123.2, 121.1, 119.6, 36.7, 33.7. HRMS (ESI) calcd for $\mathrm{C}_{22} \mathrm{H}_{17} \mathrm{ClF}_{3} \mathrm{~N}_{4} \mathrm{O}_{4} \mathrm{Na}[\mathrm{M}+\mathrm{Na}]^{+}$515.0704, found 515.0704 .

$N$-(2-(3-氯-5-(三氟甲基)吡啶-2-基)乙基)-2-(3-氯苯 甲酰胺基)苯甲酰胺(7p): 黄色固体, 收率 87\%. m.p. 136 138 ${ }^{\circ} \mathrm{C}$; ${ }^{1} \mathrm{H}$ NMR (300 MHz, $\left.\mathrm{CDCl}_{3}\right) \delta: 12.32$ (s, $1 \mathrm{H}, 8.74(\mathrm{~d}, J=7.9 \mathrm{~Hz}, 2 \mathrm{H}), 8.03(\mathrm{t}, J=1.8 \mathrm{~Hz}, 1 \mathrm{H}), 7.89$ (ddd, $J=7.5,3.9,1.6 \mathrm{~Hz}, 2 \mathrm{H}), 7.59 \sim 7.33(\mathrm{~m}, 5 \mathrm{H}), 7.15 \sim$ $7.00(\mathrm{~m}, 1 \mathrm{H}), 4.00(\mathrm{dd}, J=12.0,6.0 \mathrm{~Hz}, 2 \mathrm{H}), 3.34$ (t, $J=$ $6.0 \mathrm{~Hz}, 2 \mathrm{H}) ;{ }^{13} \mathrm{C}$ NMR $\left(75 \mathrm{MHz}, \mathrm{CDCl}_{3}\right) \delta: 168.7,163.7$, $160.5,145.9,143.4,139.5,135.5,133.7,132.5,131.6$, $131.3,130.8,129.8,129.0,128.0,126.4,125.9,125.6$, $125.3,124.6,124.1,122.9,121.7,121.2,120.5,119.8$, 36.7, 33.8. HRMS (ESI) calcd for $\mathrm{C}_{22} \mathrm{H}_{17} \mathrm{Cl}_{2} \mathrm{~F}_{3} \mathrm{~N}_{3} \mathrm{O}_{2}[\mathrm{M}+$ $\mathrm{H}]^{+}$482.0644, found 482.0651 .

$\mathrm{N}$-(2-(3-氯-5-(三氟甲基)吡啶-2-基)乙基)-2-(4-氯苯 甲酰胺基)苯甲酰胺(7q): 黄色固体, 收率 85\%. m.p. $138 \sim 140{ }^{\circ} \mathrm{C}$; ${ }^{1} \mathrm{H}$ NMR (300 MHz, $\left.\mathrm{CDCl}_{3}\right) \delta: 12.32$ (s, $1 \mathrm{H}), 8.75(\mathrm{dd}, J=4.2,3.3 \mathrm{~Hz}, 2 \mathrm{H}), 8.01 \sim 7.95(\mathrm{~m}, 2 \mathrm{H})$, $7.92(\mathrm{~d}, J=1.7 \mathrm{~Hz}, 1 \mathrm{H}), 7.50(\mathrm{t}, J=8.5 \mathrm{~Hz}, 5 \mathrm{H}), 7.11 \sim$ $7.03(\mathrm{~m}, 1 \mathrm{H}), 4.00$ (q, $J=5.9 \mathrm{~Hz}, 2 \mathrm{H}), 3.34$ (t, $J=5.9 \mathrm{~Hz}$, $2 \mathrm{H}) ;{ }^{13} \mathrm{C}$ NMR (75 MHz, $\left.\mathrm{CDCl}_{3}\right) \delta: 168.7,164.1,160.5$, 143.4, 139.6, 137.8, 133.6, 133.0, 132.4, 131.6, 128.6, 126.1, 125.6, 124.1, 122.6, 121.1, 119.8, 36.7, 33.8. HRMS (ESI) calcd for $\mathrm{C}_{22} \mathrm{H}_{17} \mathrm{Cl}_{2} \mathrm{~F}_{3} \mathrm{~N}_{3} \mathrm{O}_{2}[\mathrm{M}+\mathrm{H}]^{+}$482.0644, found 482.0651 .

$N$-(2-((2-(3-氯-5-(三氟甲基)吡啶-2-基)乙基)氨甲酰 基)苯基)-[1,1'-联苯]-4-甲酰胺(7r)：白色固体，收率 
82\%. m.p. $136 \sim 138{ }^{\circ} \mathrm{C} ;{ }^{1} \mathrm{H}$ NMR $\left(300 \mathrm{MHz}, \mathrm{CDCl}_{3}\right) \delta$ : $12.31(\mathrm{~s}, 1 \mathrm{H}), 8.82$ (d, $J=7.9 \mathrm{~Hz}, 1 \mathrm{H}), 8.76$ (d, $J=0.9 \mathrm{~Hz}$, $1 \mathrm{H}), 8.13(\mathrm{~d}, J=8.4 \mathrm{~Hz}, 2 \mathrm{H}), 7.92$ (d, $J=1.7 \mathrm{~Hz}, 1 \mathrm{H}), 7.76$ $(\mathrm{d}, J=8.4 \mathrm{~Hz}, 2 \mathrm{H}), 7.70 \sim 7.64(\mathrm{~m}, 2 \mathrm{H}), 7.56 \sim 7.46(\mathrm{~m}$, $5 \mathrm{H}), 7.43(\mathrm{~d}, J=7.2 \mathrm{~Hz}, 1 \mathrm{H}), 7.13 \sim 7.04(\mathrm{~m}, 1 \mathrm{H}), 4.02$ (q, $J=5.9 \mathrm{~Hz}, 2 \mathrm{H}), 3.35(\mathrm{t}, J=5.9 \mathrm{~Hz}, 2 \mathrm{H}) ;{ }^{13} \mathrm{C} \mathrm{NMR}(75$ MHz, DMSO) $\delta$ : 168.8, 165.0, 160.5, 144.2, 143.4, 139.8, 134.2 , 133.1, 132.3, 131.6, 128.6, 127.6, 127.0, 126.3, 126.0, 125.5, 122.5, 121.2, 120.0, 36.8, 33.8. HRMS (ESI) calcd for $\mathrm{C}_{28} \mathrm{H}_{22} \mathrm{ClF}_{3} \mathrm{~N}_{3} \mathrm{O}_{2}[\mathrm{M}+\mathrm{H}]^{+}$524.1349, found 524.1347 .

2,4-二氯- $N-(2-((2-(3-$ 氯-5-(三氟甲基)吡啶-2-基) 乙 基)氨甲酰基)苯基)苯甲酰胺(7s): 淡黄色固体, 收率 $85 \%$. m.p. $138 \sim 140{ }^{\circ} \mathrm{C}$; ${ }^{1} \mathrm{H}$ NMR $\left(300 \mathrm{MHz}, \mathrm{CDCl}_{3}\right) \delta$ : 11.75 (s, 1H), 8.73 (d, $J=8.8 \mathrm{~Hz}, 2 \mathrm{H}), 7.92$ (d, $J=1.9 \mathrm{~Hz}$, 1H), $7.60(\mathrm{~d}, J=8.3 \mathrm{~Hz}, 1 \mathrm{H}), 7.56 \sim 7.39$ (m, 4H), 7.35 $(\mathrm{dd}, J=8.3,2.0 \mathrm{~Hz}, 1 \mathrm{H}), 7.16 \sim 7.08(\mathrm{~m}, 1 \mathrm{H}), 3.94(\mathrm{dd}$, $J=11.9,5.9 \mathrm{~Hz}, 2 \mathrm{H}), 3.29(\mathrm{t}, J=5.9 \mathrm{~Hz}, 2 \mathrm{H}) ;{ }^{13} \mathrm{C} \mathrm{NMR}$ $\left(75 \mathrm{MHz}, \mathrm{CDCl}_{3}\right) \delta: 168.3,16.0,160.5,143.4,138.9$, $136.4,134.3,133.6,1323,132.0,131.6,130.0,127.14$, 126.1, 125.6, 124.1, 123.1, 121.3, 120.5, 36.6, 33.7. HRMS (ESI) calcd for $\mathrm{C}_{22} \mathrm{H}_{16} \mathrm{Cl}_{3} \mathrm{~F}_{3} \mathrm{~N}_{3} \mathrm{O}_{2} \quad[\mathrm{M}+\mathrm{H}]^{+}$ 516.0255 , found 516.0256 .

$N$-(2-(3-氯-5-(三氟甲基)吡啶-2-基)乙基)-2-(2-(4-甲 氧基苯基)乙胺基)苯甲酰胺(7t): 白色固体, 收率 $87 \%$. m.p. $142 \sim 144{ }^{\circ} \mathrm{C} ;{ }^{1} \mathrm{H}$ NMR $\left(300 \mathrm{MHz}, \mathrm{CDCl}_{3}\right) \delta$ : 11.16 $(\mathrm{s}, 1 \mathrm{H}), 8.74(\mathrm{~d}, J=1.0 \mathrm{~Hz}, 1 \mathrm{H}), 8.55(\mathrm{~d}, J=8.4 \mathrm{~Hz}, 1 \mathrm{H})$, $7.93(\mathrm{~d}, J=1.6 \mathrm{~Hz}, 1 \mathrm{H}), 7.45 \sim 7.36(\mathrm{~m}, 2 \mathrm{H}), 7.34 \sim 7.23$ $(\mathrm{m}, 3 \mathrm{H}), 7.07 \sim 6.97(\mathrm{~m}, 1 \mathrm{H}), 6.95 \sim 6.87(\mathrm{~m}, 2 \mathrm{H}), 3.92$ (dd, $J=12.0,5.9 \mathrm{~Hz}, 2 \mathrm{H}), 3.80(\mathrm{~s}, 3 \mathrm{H}), 3.66$ (s, 2H), 3.30 $(\mathrm{t}, J=6.0 \mathrm{~Hz}, 2 \mathrm{H}) ;{ }^{13} \mathrm{C} \mathrm{NMR}\left(75 \mathrm{MHz}, \mathrm{CDCl}_{3}\right) \delta: 169.9$, $168.4,160.5,158.5,143.4,139.2$, 133.6, 132.0, 131.6, $130.1,126.5,126.0,125.5,124.2,122.4,121.1,120.4$, 113.9, 54.9, 44.4, 36.6, 33.9. HRMS (ESI) calcd for $\mathrm{C}_{24} \mathrm{H}_{22} \mathrm{ClF}_{3} \mathrm{~N}_{3} \mathrm{O}_{3}[\mathrm{M}+\mathrm{H}]^{+}$492.1299, found 492.1290 .

4-氯- $N$-(2-((2-(3-氯-5-(三氟甲基)吡啶-2-基)乙基)氨 甲酰基)苯基)-2-(三氟甲基)苯甲酰胺(7u): 白色固体, 收 率 86\%. m.p. 152 $154{ }^{\circ} \mathrm{C} ;{ }^{1} \mathrm{H}$ NMR (300 $\mathrm{MHz}, \mathrm{CDCl}_{3}$ ) $\delta: 12.59(\mathrm{~s}, 1 \mathrm{H}), 8.81 \sim 8.70(\mathrm{~m}, 2 \mathrm{H}), 8.42(\mathrm{~d}, J=1.9 \mathrm{~Hz}$, $1 \mathrm{H}), 8.11(\mathrm{dd}, J=8.4,2.1 \mathrm{~Hz}, 1 \mathrm{H}), 7.94$ (d, $J=1.9 \mathrm{~Hz}$, $1 \mathrm{H}), 7.66(\mathrm{~d}, J=8.3 \mathrm{~Hz}, 1 \mathrm{H}), 7.51(\mathrm{dd}, J=8.2,6.9 \mathrm{~Hz}$, $3 \mathrm{H}), 7.13(\mathrm{~s}, 1 \mathrm{H}), 4.01(\mathrm{~d}, J=5.9 \mathrm{~Hz}, 2 \mathrm{H}), 3.35$ (t, $J=5.8$ $\mathrm{Hz}, 2 \mathrm{H}) ;{ }^{13} \mathrm{C}$ NMR $\left(75 \mathrm{MHz}, \mathrm{CDCl}_{3}\right) \delta: 168.6,162.7$, $160.5,143.4,139.5,135.5,134.3,132.6,131.61,130.7$, $128.9,128.5,127.0,126.2,125.6,124.1,123.0,121.1$,
120.4, 119.6, 36.7, 33.7. HRMS (ESI) calcd for $\mathrm{C}_{23} \mathrm{H}_{16} \mathrm{Cl}_{2} \mathrm{~F}_{6} \mathrm{~N}_{3} \mathrm{O}_{2} \mathrm{Na}[\mathrm{M}+\mathrm{Na}]^{+}$572.0338, found 572.0338 .

$N$-(2-((2-(3-氯-5-(三氟甲基)吡啶-2-基)乙基)氨甲酰 基)苯基)-3,5-二硝基苯甲酰胺(7v)：白色固体，收率 85\%. m.p. $176 \sim 178{ }^{\circ} \mathrm{C} ;{ }^{1} \mathrm{H} \operatorname{NMR}\left(300 \mathrm{MHz} \mathrm{CDCl}_{3}\right) \delta$ : $12.74(\mathrm{~d}, J=7.7 \mathrm{~Hz}, 1 \mathrm{H}), 9.00(\mathrm{~d}, J=6.2 \mathrm{~Hz}, 4 \mathrm{H}), 8.84$ (d, $J=0.9 \mathrm{~Hz}, 1 \mathrm{H}), 8.38(\mathrm{dd}, J=18.3,4.8 \mathrm{~Hz}, 2 \mathrm{H}), 7.82 \sim$ $7.72(\mathrm{~m}, 1 \mathrm{H}), 7.62 \sim 7.52(\mathrm{~m}, 1 \mathrm{H}), 7.26(\mathrm{t}, J=7.2 \mathrm{~Hz}, 1 \mathrm{H})$, 3.75 (dd, $J=12.7,6.6 \mathrm{~Hz}, 2 \mathrm{H}), 3.28$ (t, $J=6.9 \mathrm{~Hz}, 2 \mathrm{H})$; ${ }^{13} \mathrm{C}$ NMR (75 MHz, DMSO) $\delta: 168.6,161.0,160.5,148.5$, $144.0,138.2,137.4,134.2,132.3,131.2,128.4,127.2$, 124.8, 124.3, 122.1, 121.4, 37.7, 34.5. HRMS (ESI) calcd for $\mathrm{C}_{22} \mathrm{H}_{16} \mathrm{ClF}_{3} \mathrm{~N}_{5} \mathrm{O}_{6}[\mathrm{M}+\mathrm{H}]^{+}$538.0736, found 538.0727.

\section{3 生物活性测试}

\subsection{1目标化合物对秀丽隐杆线虫的杀虫活性实验}

目标化合物的杀虫活性参考文献 ${ }^{[12,20-22]}$. 准备好高 压灭菌过的 $\mathrm{NGM}$ 培养基，在培养基表面加 $150 \mu \mathrm{L}$ 大肠 杆菌 OP50, 涂抹均匀，待其晾干后将虫体放入，置于 $22{ }^{\circ} \mathrm{C}$ 恒温箱培养. $3 \mathrm{~d}$ 后取出, 用 $\mathrm{M} 9$ 缓冲液冲洗长满秀 丽线虫的培养基，将虫体收集至 $2 \mathrm{~mL}$ 离心管中，离心 弃去上清液，重复洗三次. 在离心管中加入 $2 \mathrm{~mL}$ 虫子 裂解液, 混匀, 静置 $20 \mathrm{~min}$ 后, $3000 \mathrm{r} / \mathrm{min}$ 离心, 弃去上 清液, 用 M9 缓冲液洗 3 次. 将虫卵加入新鲜涂满 OP50 的 NGM 中，置于 $22{ }^{\circ} \mathrm{C}$ 恒温箱中培养 $3 \mathrm{~d}$ 得到幼虫. 用 灭菌水冲洗幼虫, 然后用 $3800 \mathrm{r} / \mathrm{min}$ 离心处理, 弃大部 分上清溶液，收集下层溶液，检测线虫浓度后备用.

分别称取目标化合物 $1 \mathrm{mg}$, 加 $50 \mu \mathrm{L}$ DMSO 溶解, 再加入 $10 \mathrm{~mL} 1 \%$ 吐温 80 水溶液. 在 48 孔板上每个加入 $250 \mu \mathrm{L}$ 不同化合物的水溶液，在化合物浓度为 100 $\mu \mathrm{g} / \mathrm{mL}$ 的情况下测定，设置三个平行实验，以氟吡菌酰 胺为对照药剂. 每个孔中加入 $10 \mu \mathrm{L}$ 富集好的秀丽隐杆 线虫二龄幼虫水溶液, 数活的线虫个数, 然后记录初始 数值, $24 \mathrm{~h}$ 后记录存活数值, 并做空白对照. 线虫数目 需保持在 $100 \sim 200$.

\subsection{2 目标化合物的抑制菌丝生长活性实验}

本试验按照中华人民共和国农业行业标准(NY/T 1156.2-2006), 采用菌丝生长速率法进行测定 ${ }^{[23-24]}$. 用 万分之一电子天平分别称取待测化合物和氟吡菌酰胺 $20 \mathrm{mg}$, 加入到 $10 \mathrm{~mL}$ 离心管中, 用 $2 \mathrm{~mL}$ DMSO 溶解, 制备成 $1 \mathrm{mg} / \mathrm{mL}$ 的母液. 在超净无菌条件下，把配制好 的母液用培养基稀释成 $50 \mathrm{mg} / \mathrm{L}$ 的含毒培养基平板，试 验设不含药剂处理的空白对照, 各处理重复 3 次. 将活 化好的苹果腐烂菌、苹果轮纹菌、油菜菌核菌以及番茄 灰酶病原菌，在超净实验台上无菌条件下用直径为 5 
$\mathrm{mm}$ 的打孔器打孔, 切取菌饼, 用 5 号手术刀将菌饼接 种于冷却后的含药培养基的中央, 盖上血盖倒置于培养 箱中培养, 设置三个平行实验, 统计结果并取平均值.

\subsection{3 目标化合物的酶抑制活性实验}

根据参考文献[25]报道的方法提取线粒体. 将苹果 腐烂病原菌接种在马铃薯葡萄糖琼脂(PDA)培养基上, 然后 $25{ }^{\circ} \mathrm{C}$ 暗箱培养 $7 \mathrm{~d}$. 用打孔器从 PDA 培养基边缘 生长旺盛的部位打孔, 取菌饼接入马铃薯葡萄糖肉汤 (PDB)中, 摇床暗箱培养 $7 \mathrm{~d}\left(25{ }^{\circ} \mathrm{C}, 180 \mathrm{r} / \mathrm{min}\right)$. 将菌体 真空抽干其水分, 用小刀切成小块, 加入 $20 \mathrm{~mL}$ 线粒体 提取液 $\left(10 \mathrm{mmol} \cdot \mathrm{L}^{-1} \mathrm{KH}_{2} \mathrm{PO}_{4}, 10 \mathrm{mmol} \cdot \mathrm{L}^{-1} \mathrm{KCl}, 10\right.$ $\mathrm{mmol} \cdot \mathrm{L}^{-1} \mathrm{MgCl}_{2}, 0.5 \mathrm{mmol} \cdot \mathrm{L}^{-1}$ 蔗糖, $0.2 \mathrm{mmol} \cdot \mathrm{L}^{-1}$ EDTA, $2 \mathrm{mmol} \cdot \mathrm{L}^{-1}$ 苯基甲基磺酰氟(PMSF)). 用超声破 碎破碎菌体 $(200 \mathrm{~W}, 15 \mathrm{~s}$, 间隔 $20 \mathrm{~s}$, 循环 6 次), 然后离 心分离 $\left(4{ }^{\circ} \mathrm{C}, 5000 \mathrm{~g}, 10 \mathrm{~min}\right)$, 取上清液. 上清液 13000 $\mathrm{g}$ 离心 $20 \mathrm{~min}$, 弃上清液, 沉淀即为线粒体颗粒. 重新悬 浮在相同的缓冲液中. 线粒体悬浮液的浓度为 10 $\mathrm{mg} / \mathrm{mL}$ ，使用前储存于 $-80{ }^{\circ} \mathrm{C}$.

采用南京建城生物工程研究所琥珀酸脱氢酶测定 试剂盒测定化合物 7a $7 \mathbf{v}$ 的 SDH 酶抑制活性. 待测目 标化合物溶解于 DMSO 中, 以 DMSO 为对照. 线粒体悬 浮液在提取缓冲液中稀释 $1 / 10$, 并在 $10 \mathrm{mmol} \cdot \mathrm{L}^{-1}$ 琥珀 酸存在下 $30{ }^{\circ} \mathrm{C}$ 预激活 $30 \mathrm{~min}$, 将线粒体和目标化合物 共孵育 $3 \mathrm{~min}$, 在 $30{ }^{\circ} \mathrm{C}$ 下进行 DCPIP 还原, 用分光光度 计在 $600 \mathrm{~nm}$ 下监测, 测定 5 和 $65 \mathrm{~s}$ 的吸光度变化值.

\section{4 分子对接}

为了研究化合物与 $\mathrm{SDH}$ 的结合模式, 采用 Sybyl-X2.0 进行分子对接研究. 首先用 ChemBioDraw 16.0 画出目标化合物的结构, 然后用 ChemBio3D16.0 转 为化合物的三维结构, 并在 Sybyl 软件中使用 MMFF94 力场进行优化. 在 PDB 数据库(http://www.ncbi.nlm.nih. gov/protein/)下载 SDH 的晶体结构(4ysz), 在 Sybyl 软件 中进行, 通过提取配体, 消除水分子, 修复侧链, 加氢 后来构建 SDH 的活性口袋, 然后通过 Surflex-Dock 模块 进行分子对接研究, 选取最优构象, 观察分子与蛋白的 结合方式以及相互作用.

辅助材料(Supporting Information) 化合物 $7 \mathbf{a} \sim 7 \mathrm{v}$ 的 ${ }^{1} \mathrm{H}$ NMR 和 ${ }^{13} \mathrm{C}$ NMR 图谱. 这些材料可以免费从本刊网 站(http://sioc-journal. cn/)上下载.

\section{References}

[1] Chen, L.; Zhao, B.; Fan, Z.; Hu, M.; Li, Q.; Hu, W.; Li, J.; Zhang, J. J. Agric. Food Chem. 2019, 67, 12357.

[2] Silva, F. J.; Campos, V. P.; Oliveira, D. F.; Gomes, V. A.; Barros, A. F.; Din, Z. U.; Rodrigues - Filho, E. J. Phytopathol. 2019, 167, 197.

[3] Hu, Y.; Zhang, W.; Zhang, P.; Ruan, W.; Zhu, X. J. Agric. Food Chem. 2013, 61, 41 .

[4] Rao, S. V.; Sriram, K. I. J. Enzyme Inhib. Med. Chem. 1997, 11, 293.

[5] Inaoka, D. K.; Shiba, T.; Sato, D.; Balogun, E. O.; Sasaki, T.; Nagahama, M.; Oda, M.; Matsuoka, S.; Ohmori, J.; Honma, T.; Inoue, M.; Kita, K.; Harada, S. Int. J. Mol. Sci. 2015, 16, 15287.

[6] Keohane, C. E.; Steele, A. D.; Fetzer, C.; Khowsathit, J.; Van Tyne, D.; Moynie, L.; Gilmore, M. S.; Karanicolas, J.; Sieber, S. A.; Wuest, W. M.. J. Am. Chem. Soc. 2018, 140, 1774.

[7] Diaz-Quiroz, D. C.; Cardona-Felix, C. S.; Viveros-Ceballos, J. L.; Reyes-Gonzalez, M. A.; Bolivar, F.; Ordonez, M.; Escalante, A. J. Enzyme Inhib. Med. Chem. 2018, 33, 397.

[8] Liang, P.; Shen, S.; Xu, Q.; Wang, S.; Jin, S.; Lu, H.; Dong, Y.; Zhang, Bioorg. Med. Chem. 2021, 29, 115846.

[9] Li, A.; Li Z.; Zhao Y.; Yao T.; Cheng J.; Zhao J. Chin. J. Org. Chem. 2020, 40, 2836 (in Chinese).

(李安邦, 李中珊, 赵洋, 姚停停, 程敬丽, 赵金浩, 有机化学, 2020, 40, 2836.)

[10] Xiao T.; Cheng W.; Qian W.; Zhang T.; Lu T.; Gao Y.; Tang X. Chin. J. Org. Chem. 2020, 40, 1704 (in Chinese).

(肖婷婷, 程玮, 钱伟烽, 张婷婷, 陆童, 高扬, 唐孝荣, 有机化 学, 2020, 40, 1704.)

[11] Hua, X.; Liu, N.; Fan, Z.; Zong, G.; Ma, Y.; Lei, K.; Yin, H.; Wang, G. Chin. J. Org. Chem. 2019, 39, 2581 (in Chinese) (华学文, 刘南南, 范志金, 宗广宁, 马翼, 雷康, 殷吴, 王桂清, 有机化学, 2019, 39, 2581.)

[12] Chen, J.; Yi, C.; Wang, S.; Wu, S.; Li, S.; Hu, D.; Song, B. Bioorg. Med. Chem. Lett. 2019, 29, 1203.

[13] Good, J. A.; Silver, J.; Nunez-Otero, C.; Bahnan, W.; Krishnan, K. S.; Salin, O.; Engstrom, P.; Svensson, R.; Artursson, P.; Gylfe, A.; Bergstrom, S.; Almqvist, F. J. Med. Chem. 2016, 59, 2094.

[14] Wang, P.-Y.; Shao, W.-B.; Xue, H.-T.; Fang, H.-S.; Zhou, J.; Wu, Z.-B.; Song, B.-A.; Yang, S. Res. Chem. Intermed. 2017, 43, 6115.

[15] Sharma, S.; Gangal, S.; Rauf, A.; Zahin, M. Arch. Pharm. (Weinheim, Ger.) 2008, 341, 714.

[16] Nayyab, S.; O'Connor, M.; Brewster, J.; Gravier, J.; Jamieson, M.; Magno, E.; Miller, R. D.; Phelan, D.; Roohani, K.; Williard, P.; Basu, A.; Reid, C. W. ACS Infect. Dis. 2017, 3, 421.

[17] Pejchalova, M.; Havelek, R.; Kralovec, K.; Ruzickova, Z.; Pejchal, V. Med. Chem. Res. 2017, 26, 1847.

[18] Clark, D. A.; Lahm, G. P.; Smith, B. K.; Barry, J. D.; Clagg, D. G. Bioorg. Med. Chem. 2008, 16, 3163.

[19] Moradi, W.; Himmler, W.; Muller, T.; Schnatterer, A.; Schlegel, G. EP 2014074212, 2015.

[20] Chen, X.; Jia, H.; Li, Z.; Xu, X. Chin. Chem. Lett. 2019, 30, 1207.

[21] Wang, G.; Chen, X.; Deng, Y.; Li, Z.; Xu, X. J. Agric. Food Chem. 2015, 63, 6883 .

[22] Caboni, P.; Aissani, N.; Demurtas, M.; Ntalli, N.; Onnis, V. Pest Manage. Sci. 2016, 72, 125 .

[23] Tang, Z.; Li, X.; Yao, Y.; Qi, Y.; Wang, M.; Dai, N.; Wen, Y.; Wan, Y.; Peng, L. Bioorg. Med. Chem. 2019, 27, 2572.

[24] Zhang, A.; Yue, Y.; Yang, J.; Shi, J.; Tao, K.; Jin, H.; Hou, T. J. Agric. Food Chem. 2019, 67, 5008.

[25] Zeun, R.; Scalliet, G.; Oostendorp, M. Pest Manage. Sci. 2013, 69, 527.

(Li, L.; Fan, Y.) 